\title{
Radial Velocities, Space Motions, and Nearby Young Moving Group Memberships of Eleven Candidate Young Brown Dwarfs*
}

\author{
Adric R. Riedel ${ }^{1,2,3,4,5}$ (iD), Victoria DiTomasso ${ }^{4,5,6}$ (D), Emily L. Rice ${ }^{4,7,8}$ (D), Munazza K. Alam ${ }^{4,5,9}$ (iD), Ellianna Abrahams ${ }^{4,10,11}$, \\ James Crook ${ }^{4,12,13}$ (D), Kelle L. Cruz ${ }^{4,5,8}$ (D), and Jacqueline K. Faherty ${ }^{4,14}$ (iD \\ ${ }^{1}$ Space Telescope Science Institute, Baltimore, MD 21218, USA \\ ${ }^{2}$ Department of Astronomy, California Institute of Technology, Pasadena, CA 91125, USA \\ ${ }^{3}$ Department of Engineering Science and Physics, College of Staten Island, City University of New York, Staten Island, NY 10314, USA \\ ${ }^{4}$ Department of Astrophysics, American Museum of Natural History, New York, NY 10024, USA \\ ${ }^{5}$ Department of Physics and Astronomy, Hunter College, City University of New York, New York, NY 10065, USA \\ ${ }^{6}$ Leibniz-Institute for Astrophysics Potsdam (AIP), An der Sternwarte 16, D-14482 Potsdam, Germany
${ }^{7}$ Department of Physics and Astronomy, College of Staten Island, City University of New York, Staten Island, NY 10314, USA \\ ${ }^{8}$ Physics Program, The Graduate Center, City University of New York, 365 Fifth Avenue, New York, NY 10016, USA \\ ${ }^{9}$ Department of Astronomy, Harvard University, Cambridge, MA 02138, USA \\ ${ }^{10}$ Department of Astronomy, University of California at Berkeley, Berkeley, CA 94720, USA \\ ${ }^{11}$ Department of Physics, City College of New York, City University of New York, New York, NY 10031, USA \\ ${ }^{12}$ Hunter College High School, 71 East 94th Street, New York, NY 10128, USA \\ ${ }^{13}$ Physics and Astronomy Department, University of California Los Angeles, 430 Portola Plaza, Los Angeles, CA 90095, USA \\ ${ }^{14}$ Department of Terrestrial Magnetism, Carnegie Institution of Washington, DC 20015, USA \\ Received 2018 November 30; revised 2019 April 1; accepted 2019 April 13; published 2019 June 3
}

\begin{abstract}
We present new radial velocity (RV) measurements for 11 candidate young very-low-mass stars and brown dwarfs, with spectral types from M7 to L7. Candidate young objects were identified by features indicative of low surface gravity in their optical and/or near-infrared spectra. RV measurements are derived from high-resolution $(R=$ $\lambda / \Delta \lambda=20,000) J$-band spectra taken with NIRSPEC at the Keck Observatory. We combine RVs with proper motions and trigonometric distances to calculate three-dimensional space positions and motions and to evaluate membership probabilities for nearby young moving groups (NYMGs). We propose 2MASS J00452143+1634446 (L2 $\beta, J=13.06$ ) as an RV standard given the precision and stability of measurements from three different studies. We test the precision and accuracy of our RV measurements as a function of spectral type of the comparison object, finding that RV results are essentially indistinguishable even with differences of \pm 5 spectral subtypes. We also investigate the strengths of gravity-sensitive $\mathrm{K}$ I lines at $1.24-1.25 \mu \mathrm{m}$ and evaluate their consistency with other age indicators. We confirm or reconfirm four brown dwarf members of NYMGs-2MASS J00452143+1634446, WISE J00470038+6803543, 2MASS J011747483403258, and 2MASS J193555952846343 — and their previous age estimates. We identify one new brown dwarf member of the Carina-Near moving group, 2M2154-10. The remaining objects do not appear to be members of any known NYMGs, despite their spectral signatures of youth. These results add to the growing number of very-low-mass objects exhibiting signatures of youth that lack likely membership in a known NYMG, thereby compounding the mystery regarding local, low-density star formation.
\end{abstract}

Key words: brown dwarfs - infrared: stars - stars: low-mass - techniques: radial velocities - techniques: spectroscopic

\section{Introduction}

Studying brown dwarfs is our gateway to constraining the formation and evolutionary histories of giant planets and their atmospheres. Brown dwarfs, especially young objects, can have masses and temperatures comparable to directly imaged exoplanets (Liu et al. 2013), but as free-floating objects rather than as stellar companions, they are more amenable to detailed study with current instrumentation. With the current generation of high-contrast integral field spectrograph instruments such as Project 1640, the Gemini Planet Imager (GPI), and the VLT Spectro-Polarimetric High-contrast Exoplanets REsearch instrument (SPHERE; Beuzit et al. 2008; Macintosh et al. 2008; Oppenheimer et al. 2013), and soon James Webb Space Telescope (Seager et al. 2009), the question of giant planet atmospheres and their formation is an increasing focus.

\footnotetext{
* Data presented herein were obtained at the W. M. Keck Observatory, which is operated as a scientific partnership among the California Institute of Technology, the University of California, and the National Aeronautics and Space Administration. The Observatory was made possible by the generous financial support of the W. M. Keck Foundation.
}

Brown dwarfs do not achieve stable hydrogen fusion, therefore, they have no main sequence and no direct massluminosity relationship. Instead, brown dwarfs continually decrease in radius, temperature, and luminosity over time. It is thus difficult to tell the difference between brown dwarfs of different masses based on spectra alone; a young low-mass brown dwarf can have the same temperature as an old highmass brown dwarf. Indeed, for many very-low-mass objects it is not possible to determine whether an object is a star or a brown dwarf without an estimate of the object's age. There are two ways to resolve this mass-age degeneracy: dynamical mass measurement (e.g., Konopacky et al. 2010; Dupuy et al. 2014, 2015), which uses a combination of astrometry and spectroscopy to determine dynamical masses; and age measurements, which currently rely on spectroscopic and kinematic diagnostics. Dynamical masses require the brown dwarf to be in a close binary system, which is rare $\left(2.5_{-1.6}^{+8.6} \%\right.$ of the population; Blake et al. 2010), and a complete (or at least partial) orbit, which can require years to decades of astrometric monitoring. Precise age measurements for field-age and 
younger brown dwarfs (i.e., non-subdwarfs) require either a stellar companion with a reliable age constraint or membership in a nearby young moving group (NYMG), cluster, or starforming region where age constraints are then provided by the NYMG as a whole, typically based on age constraints determined using higher mass members (e.g., Zuckerman \& Song 2004).

For young, single brown dwarfs, the most readily accessible method to estimate age is via kinematic membership in an NYMG. The NYMGs are, as their name implies, groups of stars and brown dwarfs moving together through space with similar space velocities. The assumption is that they formed together in a single star-forming event, with the same Galactic orbits as their natal molecular cloud. Though they are not gravitationally bound to each other in an open cluster, they are still young enough that shear from the Galactic potential and chance encounters with disk stars have not completely obscured their shared trajectory. As such, determining the space velocity (and space position) of young objects is a powerful method of determining their potential membership in an NYMG. NYMGs are sparse, containing perhaps a few hundred members spread out over thousands of cubic parsecs. Known groups include $\beta$ Pictoris ( 20 Myr; Mamajek \& Bell 2014), Tucana-Horologium ( 45 Myr; Bell et al. 2015), Argus ( 50 Myr; Barrado y Navascués et al. 1999), and AB Doradus ( 120 Myr; Binks \& Jeffries 2014; Bell et al. 2015). NYMGs are windows into the later stages of star and planetary system formation. At these ages, natal gas and dust are dissipated, removing extinction within the brown dwarf system, but brown dwarfs and very-low-mass stars are still physically enlarged compared to their field ( $>1 \mathrm{Gyr}$ ) equivalents. Thus they can exhibit spectral signatures of low surface gravity and potentially have different atmospheric cloud conditions and weather patterns (e.g., Lew et al. 2016).

Probabilities of membership for individual objects in NYMGs are optimally calculated with complete spatial and velocity information, i.e., position, distance, proper motion, and radial velocity (RV). While it is possible to determine memberships with only partial kinematics, Riedel et al. (2017) demonstrate the importance of having better and more complete data. As shown in that paper, a brown dwarf can at best be given a $40 \%$ probability of membership in $\beta$ Pictoris given only proper motion information; by that same token, the maximum probability rises to over $90 \%$ with the addition of RV information, even without a distance. Age constraints provided by NYMG membership can range from $5 \mathrm{Myr}(\epsilon$ Chamæleon; Murphy et al. 2013) to $500 \mathrm{Myr}$ ( $\chi^{01}$ For; Pöhnl \& Paunzen 2010) with uncertainties of \pm 10 Myr for TW Hydra (Weinberger et al. 2013) to $\pm 100 \mathrm{Myr}$ for older groups.

The established memberships of NYMGs are deficient in low-mass members (mid-M dwarfs and later) relative to the field initial mass function (e.g., Jeffries 2012; Kraus et al. 2014; Gagné et al. 2017; Shkolnik et al. 2017). In order to complete the low-mass census of NYMGs, candidate young, very-lowmass objects are typically identified based on near-infrared (NIR) colors and low-resolution spectral features indicative of low surface gravity. Young very-low-mass objects are typically 1-2 mag redder than the average NIR color for their spectral type (Faherty et al. 2012). Spectra of these unusually red objects often exhibit spectroscopic signatures of low gravity, including weaker singly ionized alkali metal lines, which is often taken to be a sign of youth (e.g., Cruz et al. 2009). These objects are assumed to be young, with spectral type suffixes coarsely defined according to the divergence of gravitysensitive spectral features from those of field (i.e., old) objects (Cruz et al. 2009; Allers \& Liu 2013). Finer age estimation based on spectral features alone is not currently possible, therefore, establishing membership in an NYMG is essential to providing age constraints for very-low-mass objects.

There are currently over 160 objects with spectral types M7 and later that have been identified as candidate members of NYMGs. Prominent early examples included TWA 27 (2MASS J12073346-3932539, hereafter 2M1207-39) in TW Hydra (Gizis 2002), 2MASS J01415823-4633574 (hereafter 2M0141-46; Kirkpatrick et al. 2006) in TucanaHorologium (Gagné et al. 2015a), 2MASS J06085283 -2753583 (hereafter 2M0608-27) in $\beta$ Pictoris (Rice et al. 2010, but see also Gagné et al. 2014c; Faherty et al. 2016), and 2MASS J03552337+1133437 (hereafter 2M0355+11) in AB Doradus (Faherty et al. 2013; Liu et al. 2013). These objects have frequently been used as comparison objects for newly discovered candidate young low-mass objects and even directly imaged exoplanets (e.g., Crepp et al. 2018; Greenbaum et al. 2018; Miles et al. 2018). There are additionally over 150 very-low-mass stars and brown dwarfs that display signatures of youth but lack complete kinematic information (e.g., Gagné et al. 2014b, 2014c, 2015b, 2015c; Faherty et al. 2016).

Of the spatial and kinematic data required for evaluating NYMG membership, RV and parallax are arguably the most challenging measurements for intrinsically faint low-mass targets. Multiple parallax programs (e.g., Marocco et al. 2013; Dieterich et al. 2014; Zapatero Osorio et al. 2014; Faherty et al. 2016; Liu et al. 2016; Gaia Collaboration et al. 2018) are tackling the problem of parallaxes, which leaves RVs as the final important piece of the kinematic puzzle. RVs for low-mass objects necessitate either long exposure times to obtain sufficiently high-resolution and signal-to-noise ratio $(\mathrm{S} / \mathrm{N})$ spectra for RV measurements, even on the Keck $10 \mathrm{~m}$ telescope (e.g., Blake et al. 2010; Prato et al. 2015). Further, absolute RV measurements are optimally calibrated against high-quality spectra of similar spectral type objects with existing RV measurements, which are only recently beginning to exist in large enough numbers to evaluate the dependence of measured RV on spectral type, $\mathrm{S} / \mathrm{N}$, and other properties of the comparison object's spectrum.

In this paper we present new high-resolution NIR spectroscopy, obtained with NIRSPEC on Keck II, of 11 candidate young late-M and L dwarfs. We measure RVs to derive threedimensional space positions and motions for the sample, and use them to determine membership, and therefore ages, in NYMGs. Unlike similar studies that use the $K$ band (Blake et al. 2010) or the $H$ band (Faherty et al. 2016), we focus on the $J$ band, which contains numerous water absorption lines, prominent bandheads of $\mathrm{FeH}$, and regions that are largely free of telluric absorption that can be used for cross-correlated RV measurements (Prato et al. 2015). The $J$ band also contains strong alkali metal lines that are sensitive to surface gravity (e.g., McLean et al. 2007; Rice et al. 2010). The objects in our sample could be very-low-mass stars or brown dwarfs, depending on their ages, but we refer to them as brown dwarfs for the sake of simplicity.

In Section 2 we describe our sample of $11 \mathrm{M}$ and L dwarfs, the NIRSPEC/Keck II observations, and the data reduction procedure. In Section 3, we describe the analysis and results, 


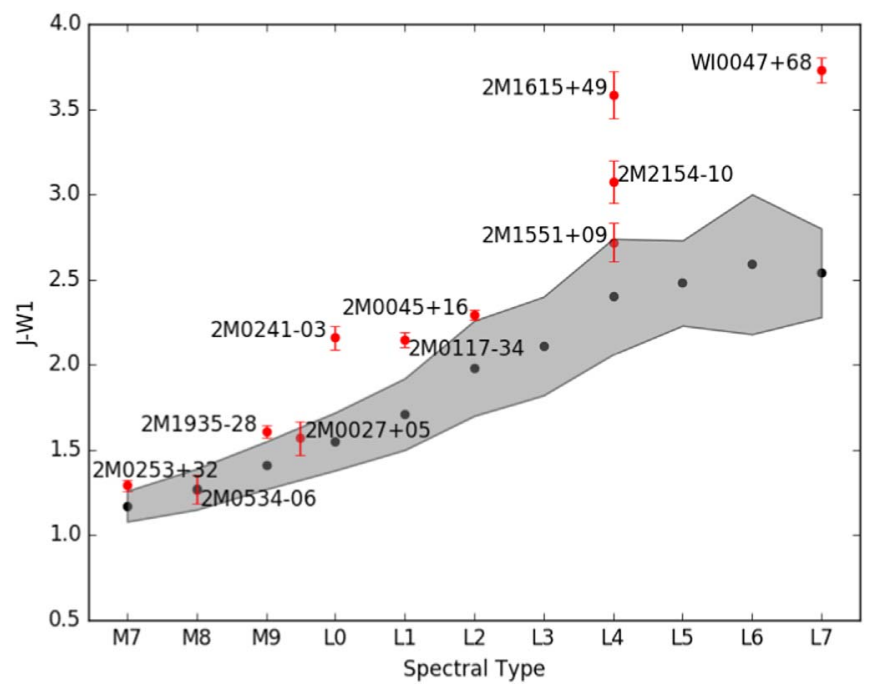

Figure 1. Spectral type vs. $J-W 1$ color diagram for the 11 sample objects (red points) plotted with the average $J-W 1$ colors (black points) and $1 \sigma$ spreads (gray shaded region) from Faherty et al. (2016). Eight of the 11 sample objects are more than $1 \sigma$ redder in $J-W 1$ color than the average for their spectral type, especially the $\mathrm{L}$ dwarfs. The remaining three are more than $1 \sigma$ red in other color combinations.

including RV measurements, the calculation of space positions and motions, and the five methods for estimating NYMG membership probabilities. We present notes on the results for individual objects in Section 4. In Section 5, we discuss the implications of our results for measuring the RV of late-type objects and for evaluating various youth indicators. We present our conclusions in Section 6.

\section{Sample, Observations, and Data Reduction}

\subsection{Sample Selection}

Our targets were selected from a sample of $\sim \mathrm{M} 7$ and later dwarfs identified as candidate young objects via their their classification as low-surface-gravity objects by Cruz et al. (2009) using red-optical spectra and/or Allers \& Liu (2013) using low-resolution, NIR spectra. All of the objects also have unusually red NIR colors for their spectral type (though not all in the specific $J-W 1$ color shown in Figure 1). Eleven objects were observed during four half-nights in 2014 March and September; details of the observations are described in Section 2.2 below and in Table 1 .

There are only minor discrepancies between the optical spectra and the NIR spectroscopy seen here. The largest differences in spectral classification is 2MASS J024111510326587 (hereafter 2M0241-03), which is an L0 $\gamma$ object by optical spectral typing (Faherty et al. 2016) but an L1 VL-G by infrared spectral typing (Allers \& Liu 2013), and with 2MASS J02535980+3206373 (hereafter $2 \mathrm{M} 0253+32$ ), which was a M7 $\beta$ by optical typing (Faherty et al. 2016) but was assigned as an M6 FLD-G in Allers \& Liu (2013).

All of these targets appear in Faherty et al. (2016), where eight of them were identified as having kinematics that suggested possible membership in multiple NYMGs or that could not be distinguished from field objects (ambiguous member). Two targets were determined by Faherty et al. (2016) to be bona fide group members: 2MASS J00452143+1634446 (hereafter $2 \mathrm{M} 0045+16$ ), identified as an Argus member by
Gagné et al. (2014c), and WISE J004701.09+680352.2 (hereafter W0047+68), identified as an AB Doradus member by Gizis et al. (2015) and Liu et al. (2016). 2MASS 01174748 -3403258 (hereafter 2M0117-34) was listed as a highlikelihood member of Tucana-Horologium by both Faherty et al. (2016) and Liu et al. (2016). Faherty et al. (2016) presented RV measurements for three of our targets, one of which (2MASS J00452143+1634446, hereafter 2M0045+16) was also previously measured by Blake et al. (2010). The other two were measured from low-quality spectra, motivating our decision to observe them again. In the time between our observations and this publication, Gizis et al. (2015) reported an $\mathrm{RV}$ for $\mathrm{W} 0047+68$. These literature RV measurements are presented and compared to our RV results in Section 3.1.

\subsection{Observations}

Observations were made UT 2014 May 22 and 24 and UT 2014 September 16 and 17 using NIRSPEC, the cryogenic cross-dispersed echelle spectrometer on the Keck II $10 \mathrm{~m}$ telescope at the W. M. Keck Observatory on Maunakea, Hawai'i (McLean et al. 1998, 2000). We used NIRSPEC's cross-dispersed echelle mode with the NIRSPEC-3 (N3) filter, which approximates the standard $J$-band coverage (1.143$1.375 \mu \mathrm{m})$. In echelle mode, eight usable dispersion orders (65-58) are captured on the detector. Because the spectral interval captured by the detector is slightly smaller than the free spectral range in each order, there are small gaps, increasing with wavelength, in the total spectral coverage. The exact wavelength ranges for each dispersion order are listed in the headings in Section 2.4. The slit width is three pixels (0." 432) for echelle observations. The resolving power in the $J$ band is approximately $R=\lambda / \Delta \lambda=20,000$ (high resolution) in echelle mode. Throughout the paper the high-resolution $J$ band spectra are referred to by the number of the dispersion order, from $65(\sim 1.17 \mu \mathrm{m})-58(\sim 1.31 \mu \mathrm{m})$.

Observing methods follow those described in detail by McLean et al. (2007) and Prato et al. (2015); the following is a brief summary and explanation of departures from those methods. Observations were made in pairs, nodding along the slit between each integration so that traces were separated by $7^{\prime \prime}$ on the $12^{\prime \prime}$-long slit. Due to a desire to avoid an intermittent quadrant in the slit-viewing camera, recent high-resolution observations have used a smaller nod length. During these occasions the nod size was at least $2^{\prime \prime}$ so that the dispersed traces would be well separated on the slit. Integration time was $600 \mathrm{~s}$ per nod for all observations except for all four exposures of $2 \mathrm{M} 0253+32$ and four (of eight total) exposures of 2MASS J05341594-0631397 (2M0534-06), which were $480 \mathrm{~s}$ per nod.

Total integration times per object are listed in Table 1 . A0 V stars were observed at an airmass very close to that of the target object (typically $<0.1$ airmass difference) to allow calibration for telluric absorption features. Arc lamp spectra were obtained at least once per night, and white-light spectra and corresponding dark frames were obtained for flat fielding.

\subsection{Data Reduction}

All of the observed data were reduced with the REDSPEC IDL-based software package, ${ }^{15}$ described in McLean et al. (2003, 2007). The package performs standard bad pixel

\footnotetext{
15 http://www2.keck.hawaii.edu/inst/nirspec/redspec.html
} 
Table 1

Observing Log

\begin{tabular}{|c|c|c|c|c|c|c|c|c|c|c|}
\hline $\begin{array}{l}\text { Object } \\
\text { Name }^{a}\end{array}$ & $\begin{array}{l}\text { 2MASS } \\
\text { ID }\end{array}$ & $\begin{array}{c}\text { Optical } \\
\text { Sp. Type }\end{array}$ & $\begin{array}{c}\text { NIR } \\
\text { Sp. Type }\end{array}$ & $\begin{array}{l}\text { Sp. Type } \\
\text { Reference }\end{array}$ & $\begin{array}{c}\alpha \\
\mathrm{J} 2000.0\end{array}$ & $\begin{array}{c}\delta \\
\mathrm{J} 2000.0\end{array}$ & $\begin{array}{c}J^{\mathrm{b}} \\
(\mathrm{mag})\end{array}$ & $\begin{array}{l}\text { Int. Time } \\
\text { (s) }\end{array}$ & $\begin{array}{c}\text { Average } \\
\mathrm{S} / \mathrm{N}\end{array}$ & $\begin{array}{l}\text { UT Date of } \\
\text { Observation }\end{array}$ \\
\hline $2 \mathrm{M} 0253+32$ & $02535980+3206373$ & $\mathrm{M} 7 \beta$ & M6 FLD-G & 1,2 & 025359.70 & +320637.0 & 13.62 & 1920 & 21 & 2014 Sep 16 \\
\hline 2M0534-06 & $05341594-0631397$ & M8 $\gamma$ & M8 VL-G & 2,1 & 053415.94 & -063139.7 & 16.05 & 4320 & 5.4 & 2014 Sep 17 \\
\hline 2M1935-28 & $19355595-2846343$ & M9 $\gamma$ & M9 VL-G & 2,1 & 193555.96 & -284634.4 & 13.95 & 3600 & 25 & 2014 May 22 \\
\hline $2 \mathrm{M} 0027+05$ & $00274197+0503417$ & M9.5 $\beta$ & L0 INT-G & 2,1 & 002741.97 & +050341.7 & 16.19 & 4800 & 5.0 & 2014 Sep 16 \\
\hline $2 \mathrm{M} 0241-03$ & $02411151-0326587$ & $\mathrm{~L} 0 \gamma$ & L1 VL-G & 3,1 & 024111.50 & -032658.0 & 15.80 & 4800 & 7.5 & 2014 Sep 16 \\
\hline $2 \mathrm{M} 0117-34$ & $01174748-3403258$ & $\mathrm{~L} 1 \beta$ & L1 INT-G & 2,1 & 011747.40 & -340325.0 & 15.18 & 4800 & 10 & 2014 Sep 17 \\
\hline $2 \mathrm{M} 0045+16$ & $00452143+1634446$ & $\mathrm{~L} 2 \beta$ & L2 VL-G & 3,1 & 004521.43 & +163444.6 & 13.06 & 1200 & 38 & 2014 Sep 16 \\
\hline $2 \mathrm{M} 1551+09$ & $15515237+0941148$ & $\mathrm{~L} 4 \gamma$ & L4 VL-G & 2,1 & 155152.37 & +094114.8 & 16.32 & 7200 & 7.3 & 2014 May 24 \\
\hline $2 \mathrm{M} 1615+49$ & $16154255+4953211$ & $\mathrm{~L} 4 \gamma$ & L3 VL-G & 2,1 & 161542.50 & +495321.0 & 16.79 & 7200 & 4.5 & 2014 May 22 \\
\hline 2M2154-10 & $21543454-1055308$ & $\mathrm{~L} 4 \beta$ & $\mathrm{L} 5 \gamma$ & 4,2 & 215434.50 & -105530.0 & 16.44 & 5400 & 4.3 & 2014 May 24 \\
\hline W0047+68 & $00470038+6803543$ & L7 $(\gamma ?)$ & L7.5 pec & 2,5 & 004701.06 & +680352.1 & 15.60 & 4800 & 6.9 & 2014 Sep 17 \\
\hline
\end{tabular}

Notes.

a 2MASS, DENIS, and SDSS object names are truncated in subsequent tables and in the text.

${ }^{\mathrm{b}}$ From 2MASS All-Sky Point Source Catalog.

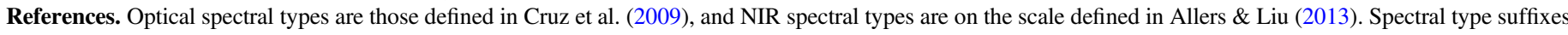

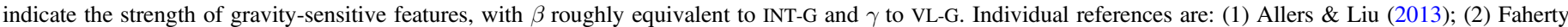
et al. (2016); (3) Cruz et al. (2009); (4) Gagné et al. (2014b); and (5) Gizis et al. (2012).

interpolation, dark subtraction, and flat fielding as well as spatial rectification of curved spectra. Spectra are rectified and extracted in subtracted nod pairs so that the sky background and $\mathrm{OH}$ emission lines are removed. Spectra were extracted by summing over 7-15 rows dependent on seeing, then subtracted again to produce a positive spectrum with residual sky emission features removed. Most orders were wavelength calibrated with $\mathrm{OH}$ night sky lines, which were found to be highly stable and well distributed across orders. Seven highresolution dispersion orders were reduced, covering orders 58-65 with the exception of order 60, where the $\mathrm{OH}$ night sky lines are blended with $\mathrm{O}_{2}$ emission bands at 1.26-1.28 $\mu \mathrm{m}$ (Rousselot et al. 2000) making wavelength calibration and sky subtraction considerably more difficult. Each reduced spectrum was continuum normalized, and multiple nod pairs were averaged together to increase $\mathrm{S} / \mathrm{N}$. Spectra were shifted to the heliocentric reference frame using barycentric corrections calculated using JSkyCalc. ${ }^{16}$

\subsection{Spectral Orders}

We present all reduced NIRSPEC dispersion orders for $2 \mathrm{M} 0045+16$ (L2 $\beta, J=13.06)$ in Figure 2. We summarize the relevant absorption features apparent in high-resolution $\mathrm{M}$ - and L-dwarf spectra by the NIRSPEC dispersion order below. More details can be found in McLean et al. (2007) and Rice et al. (2010).

Order $58(1.30447-1.32370 \mu \mathrm{m})$ - $\mathrm{Al}$ I doublet in the center of the order.

Order $59(1.28262-1.30151 \mu \mathrm{m})$-weak Fe I lines.

Order $60 \quad(1.26137-1.27999 \mu \mathrm{m})$-not reduced (see Section 2.3 above).

Order $61(1.24081-1.25913 \mu \mathrm{m})-\mathrm{K}$ I lines, higher $\mathrm{S} / \mathrm{N}$ than order 65 .

Order $62(1.22093-1.23899 \mu \mathrm{m})$ - used for RV measurements because of $\mathrm{FeH}$ and $\mathrm{H}_{2} \mathrm{O}$, strong well-spaced $\mathrm{OH}$ night sky lines, and weak telluric lines.

\footnotetext{
${ }^{16}$ http://www.dartmouth.edu/ physics/labs/skycalc/flyer.html
}
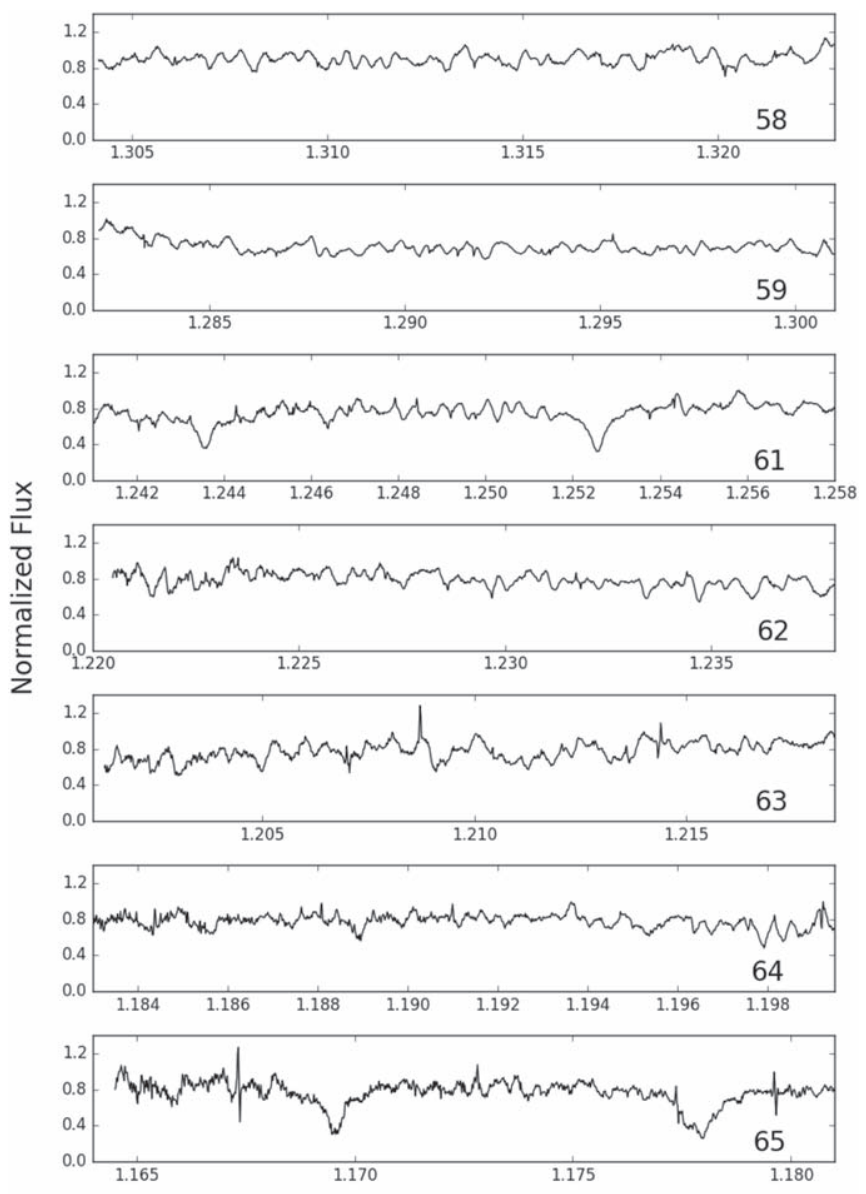

Wavelength $(\mu m)$

Figure 2. NIRSPEC spectra for dispersion orders 58,59 , and 61 to 65 for the L2 object 2M0045+16.

Order $63(1.20168-1.21938 \mu \mathrm{m})-\mathrm{FeH}$ and $\mathrm{H}_{2} \mathrm{O}$ similar to order 62.

Order $64(1.18293-1.20011 \mu \mathrm{m})$ —weak Ti I and Mn I lines. 


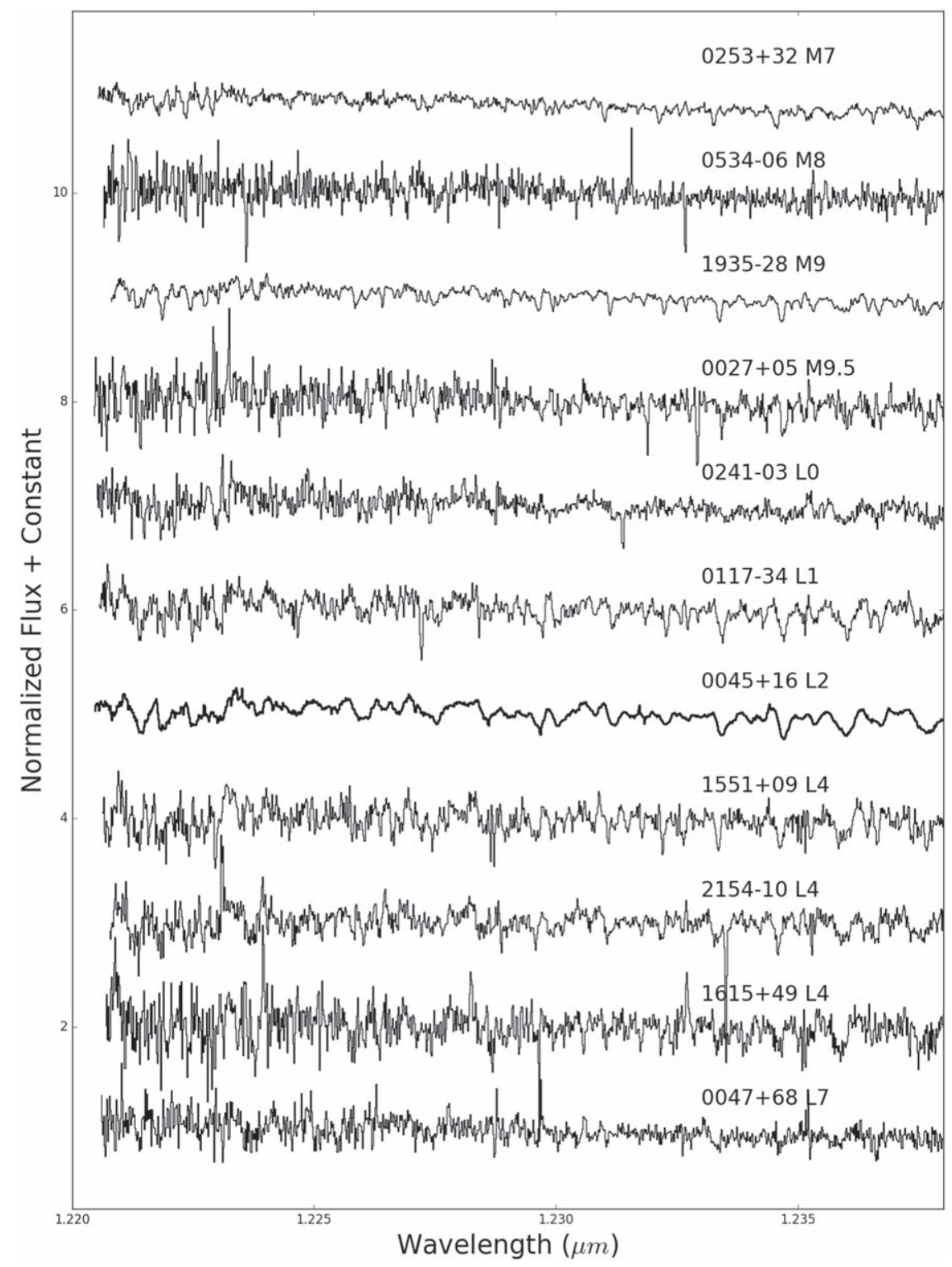

Figure 3. NIRSPEC dispersion order 62 spectra $(1.22093-1.23899 \mu \mathrm{m})$ for 11 objects in the sample.

Order 65 (1.16496-1.18207 $\mu \mathrm{m})$ - K I lines, typically lower $\mathrm{S} / \mathrm{N}$ than in order 61 and blended with $\mathrm{H}_{2} \mathrm{O}$ lines.

Following the work of the NIRSPEC Brown Dwarf Spectroscopic Survey (BDSS; Prato et al. 2015) we use order $62(1.221-1.239 \mu \mathrm{m})$ for our RV measurements. This wavelength regime is essentially free of telluric features, and $\mathrm{M}$ - and L-dwarf spectra contain numerous molecular absorption lines from $\mathrm{FeH}$ and $\mathrm{H}_{2} \mathrm{O}$ that are ideal for cross-correlation techniques. Figure 3 presents the order 62 spectra for all 11 objects in the sample. Our targets have a brightnesses between $J=13$ and $J=17$. Even with total integration times of 20 minutes to two hours (listed in Table 1), the resulting spectra have an average $\mathrm{S} / \mathrm{N}$ in order 62 between 4 and 38 , with a maximum $\mathrm{S} / \mathrm{N}$ of 38.2 for $2 \mathrm{M} 0045+16(J=13.06)$ and minimum $\mathrm{S} / \mathrm{N}$ of 4.3 for $2 \mathrm{M} 2154-10(J=16.44)$.

We also tested RV measurements using order 59, which is free of strong telluric absorption and was used by Prato et al. (2015) for cross-correlating spectra of T dwarfs. For M and L dwarfs, the intrinsic atomic lines and molecular absorption lines at these wavelengths are weaker and the results were far less reliable. No order 59 results are presented in this paper.

\section{Analysis}

\subsection{RV Measurements}

To measure RV, we use a custom cross-correlation code written in Python, first described in Faherty et al. (2016). The inputs are a heliocentric-corrected stellar spectrum (wavelength, flux, and uncertainty) and the spectrum of a comparison object with a previously measured RV, taken with the same instrumental setup to avoid systematics. For comparison spectra, we use objects with NIRSPEC BDSS spectra from McLean et al. (2007), Rice et al. (2010), and Prato et al. (2015). For the RVs of these standards, we use values reported in Rice et al. (2010), Blake et al. (2010), Chubak et al. (2012), and Prato et al. (2015), listed in Table 2.

The target and standard spectra are read in and interpolated onto a log-normal spaced wavelength grid covering only the 
Table 2

Comparison Stars

\begin{tabular}{lcccc}
\hline \hline Name & $\begin{array}{c}\text { Spectral } \\
\text { Type }\end{array}$ & $\begin{array}{c}\text { RV } \\
\left(\mathrm{km} \mathrm{s}^{-1}\right)\end{array}$ & $\begin{array}{c}\text { RV } \\
\text { References }\end{array}$ & $\begin{array}{c}\text { Average } \\
\text { S/N }\end{array}$ \\
\hline GJ 406 & M6 & $19.321 \pm 0.145$ & 1 & 326 \\
DENIS-P J1605-24 & M6 & $-5 \pm 2$ & 2 & 41 \\
SCH J1612-20 & M6.5 & $-7 \pm 2$ & 2 & 33 \\
LP 402-58 & M7 & $-3 \pm 2$ & 2 & 65 \\
UScoCTIO 130 & M7.5 & $-7 \pm 2$ & 2 & 23 \\
LP 412-31 & M8 & $42 \pm 2$ & 2 & 118 \\
2MASS & M8 & $8 \pm 2$ & 2 & 38 \\
$\quad$ J1207-39AB & & & & \\
2MASS J0608-27 & M8.5 & $23 \pm 2$ & 2 & 32 \\
2MASS 0140+27 & M8.5 & $9 \pm 2$ & 3 & 87 \\
2MASS 0345+25 & L0 & $6 \pm 3$ & 3 & 29 \\
2MASS 0746+20 & L0.5 & $52.37 \pm 0.06$ & 4 & 98 \\
2MASS 0208+25 & L1 & $20 \pm 2$ & 3 & 14 \\
2MASS 2057-02 & L1.5 & $-24.68 \pm 0.43$ & 4 & 45 \\
2MASS 0015+35 & L2 & $-37.35 \pm 0.16$ & 4 & 49 \\
2MASS 2104-10 & L2.5 & $-21.09 \pm 0.12$ & 4 & 38 \\
G 196-3B & L3 $\beta$ & $-2 \pm 2$ & 3 & 8 \\
2MASS 0036+18 & L3.5 & $19.02 \pm 0.15$ & 4 & 86 \\
2MASS 2224-01 & L4.5 & $-37.55 \pm 0.09$ & 4 & 26 \\
2MASS 1507-16 & L5 & $-39.85 \pm 0.05$ & 4 & 76 \\
\hline
\end{tabular}

Note.

${ }^{\mathrm{a}}$ Binary.

References. (1) Chubak et al. (2012); (2) Rice et al. (2010); (3) Prato et al. (2015); and (4) Blake et al. (2010).

region where the spectra overlap, up-sampled in wavelength by a factor of 10. A third-order fit to the spectra is removed, taking out the large-scale structure of the spectra and leaving only the spectral lines. The code then resamples each flux point of the target and comparison spectra from within the estimated noise on each, which we model as Gaussian random noise. The resulting resampled target spectrum is cross-correlated with the resampled RV comparison spectrum, and the cross-correlation results are fit with a Gaussian+linear function to determine the velocity shift in pixels. The process of resampling the noise is repeated 1000 times, producing 1000 velocity shift measurements for each target and comparison object spectra pair, which we bin into a histogram. The histogram of velocity measurements is fit with a Gaussian function, the mean of which we adopt as the velocity shift, and the $1 \sigma$ width of which we adopt as the uncertainty on the measurement, accounting for the noise of both spectra. The result then is converted from pixel shifts to velocity in $\mathrm{km} \mathrm{s}^{-1}$, and the known velocity of the comparison object is then subtracted to provide the actual heliocentric RV of the target. The final uncertainty of each RV measurement is the combination of the uncertainty from the cross-correlation procedure and the uncertainty in the previously measured RV of the comparison object, added in quadrature. The latter dominates the precision of the results.

This cross-correlation technique is subject to comparison object-dependent systematic errors, including uncertainties on the wavelength solution of each observation and on the previously measured RV of the comparison object. To test the accuracy of our RV results, we cross-correlate our targets with all 19 comparison objects in Table 2 using spectra from Prato et al. (2015) and Rice et al. (2010), producing 19 individual RV measurements for each of our target objects. The mean, weighted by the calculated uncertainty, of these individual RV results constitutes the final calculated RV for each target object, as listed in Table 3. This procedure was used to calculate the RVs of 8 out of 11 of our target objects.

The remaining RVs of the three objects were calculated with slight modification to the routine described above. For these three targets, the cross-correlation process produced outlying pixel shifts that implied unrealistic velocities. For those three objects, W0047+68, 2M0534-06, and 2M0241-03, we only retain pixel shifts within plausible velocity ranges (between \pm 500 or \pm 100 resampled wavelength pixels, depending on object), and run the Monte Carlo iterations until 1000 pixel shifts have been generated in that range.

For W0047+68, 15 of the 19 comparison spectra produced outlying pixel shifts. For the comparison spectra that produced outlying pixel shifts, it took an average of 1155 iterations to produce 1000 acceptable pixel shifts; the maximum was 2730 and the minimum was 1001 .

For 2M0241-03, 6 of the 19 comparison objects produced outlying pixel shifts. For two comparison objects, SCH J1612 -20 and DENIS-P J1605-24, restricting the acceptable pixel shifts to ranges of \pm 50 or fewer resampled wavelength pixels still failed to produce a Gaussian distribution of measured RVinduced pixel shifts, so we omitted these two comparison objects in the calculation of the final RV for 2M0241-03. For the other four comparison objects, it took an average of 1087 iterations to produce 1000 acceptable pixel shifts; the maximum number of iterations was 1206 and the minimum was 1008.

For 2M0534-06, all of the comparison spectra produced outlying pixel shifts. We produced Gaussian results by restricting the allowed pixel shifts for all comparison spectra. It took an average of 6236 iterations to produce 1000 acceptable pixel shifts; the maximum was 59,794 and the minimum was 1015 .

\subsection{Space Positions and Motions}

As of yet, no single photometric or spectroscopic youth indicator can be used to assign a precise and reliable age estimate for low-mass stars and brown dwarfs. Thus, we rely on kinematics—positions in R.A. and decl. (hereafter $\alpha, \delta$, and $\pi$ ); motions in $\mu_{\text {R.A.cos decl. }}, \mu_{\text {decl. }}$ (hereafter $\mu_{\alpha *}, \mu_{\delta}$ and RV)—to determine if the brown dwarfs are likely members of an NYMG. Even so, kinematics are necessary but not sufficient to prove youth. The sheer number of disk stars and the large kinematic space occupied by these unbound groups mean field-age disk stars can be interlopers, hence the importance of spectroscopic indications that the brown dwarfs are in fact young.

There are two basic strategies for determining membership in moving groups. One is to take the three positional observables $(\alpha, \delta$, and $\pi)$ and three velocity observables $\left(\mu_{\alpha *}, \mu_{\delta}, \mathrm{RV}\right)$, convert them into three-dimensional cartesian space positions $(X Y Z$, where $X$ is toward Galactic center, $Y$ is in the direction of solar motion, and $Z$ is toward the north Galactic pole) and three-dimensional cartesian space velocities $(U V W$, where $U$ is motion along the $\mathrm{x}$-axis, $V$ along the $y$-axis, and $W$ along the $z$-axis), and compare the star's $U V W X Y Z$ values to those of the moving group, represented as ellipsoids from Riedel et al. (2017) in Figures 4 and 5. The other method is to reverse the process: take the $U V W X Y Z$ properties of the moving group, translate them to observable quantities like $\mu, \mathrm{RV}$, and $\pi$ at the $\alpha$ and $\delta$ of the target star, and compare the predicted values of a group member to the actual values of the target star. 
Table 3

Astrometric and RV Data

\begin{tabular}{|c|c|c|c|c|c|c|c|c|c|c|c|c|c|c|}
\hline Name & $\begin{array}{l}\alpha \\
\left(^{\circ}\right)\end{array}$ & $\begin{array}{l}\delta \\
\left(^{\circ}\right)\end{array}$ & Reference & 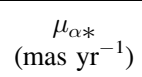 & $\begin{array}{c}\sigma_{\mu_{\alpha *}} \\
\left(\operatorname{mas~yr}^{-1}\right)\end{array}$ & 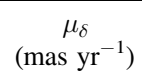 & $\begin{array}{c}\sigma_{\mu_{\delta}} \\
\left(\operatorname{mas~yr}^{-1}\right)\end{array}$ & Reference & $\begin{array}{c}\pi \\
(\mathrm{mas})\end{array}$ & $\begin{array}{c}\sigma_{\pi} \\
\text { (mas) }\end{array}$ & Reference & $\begin{array}{c}\mathrm{RV} \\
\left(\mathrm{km} \mathrm{s}^{-1}\right)\end{array}$ & $\begin{array}{c}\sigma_{\mathrm{RV}} \\
\left(\mathrm{km} \mathrm{s}^{-1}\right)\end{array}$ & Reference \\
\hline \multirow{5}{*}{$2 \mathrm{M} 0253+32$} & 43.499173 & 32.110363 & (1) & 87 & 10 & -96 & 10 & (9) & 17.7 & 2.5 & (9) & -35.114 & 1.501 & (3) \\
\hline & & & & 89.1 & 7.2 & -98.3 & 8.5 & (10) & 20.22 & 0.18 & (8) & & & \\
\hline & & & & 95.9 & 1.1 & -96.9 & 1.1 & (5) & 21.3 & 1.0 & (5) & & & \\
\hline & & & & 92.49 & 0.38 & -100.23 & 0.26 & (8) & & & & & & \\
\hline & & & & 92.84 & 0.36 & -100.05 & 0.25 & & 20.24 & 0.18 & & -35.11 & 1.50 & \\
\hline \multirow[t]{3}{*}{ 2M0534-06 } & 83.566445 & -6.52772 & (1) & 2 & 12 & -7 & 12 & (9) & & & & 28.635 & 2.938 & (3) \\
\hline & & & & 2.2 & 19.7 & -6.9 & 20.8 & (10) & & & & & & \\
\hline & & & & 2.05 & 10.25 & -6.98 & 10.39 & & & & & 28.64 & 2.94 & \\
\hline \multirow[t]{5}{*}{ 2M1935-28 } & 293.983154 & -28.776211 & (1) & 34 & 12 & -58 & 12 & (9) & 17.69 & 0.49 & (8) & -7.736 & 1.278 & (3) \\
\hline & & & & 27.3 & 0.9 & -61.6 & 1.1 & (5) & 14.2 & 1.2 & (5) & -5.08 & 3.48 & (19) \\
\hline & & & & 27.2 & 4.8 & -56.6 & 5.1 & (10) & & & & & & \\
\hline & & & & 26.40 & 0.60 & -62.09 & 0.54 & (8) & & & & & & \\
\hline & & & & 26.70 & 0.50 & -61.94 & 0.48 & & 17.19 & 0.45 & & -7.42 & 1.20 & \\
\hline \multirow[t]{3}{*}{$2 \mathrm{M} 0027+05$} & 6.924889 & 5.0616 & (1) & 10.5 & 0.4 & -0.8 & 0.3 & (2) & 13.8 & 1.6 & (2) & 6.788 & 1.541 & (3) \\
\hline & & & & 16.1 & 1.1 & -0.33 & 1.2 & (5) & 10.4 & 0.8 & (5) & & & \\
\hline & & & & 11.15 & 0.38 & -0.77 & 0.29 & & 11.08 & 0.72 & & 6.79 & 1.54 & \\
\hline \multirow[t]{6}{*}{$2 \mathrm{M} 0241-03$} & 40.297996 & -3.449661 & (1) & 73.7 & 1 & -24.2 & 1.9 & (9) & 26.7 & 3.3 & (9) & 10.221 & 2.12 & (3) \\
\hline & & & & 93.43 & 17 & -19.87 & 13.4 & (14) & 21.4 & 2.6 & (4) & 6.34 & 7.98 & (9) \\
\hline & & & & 69.6 & 0.5 & -25.1 & 0.6 & (5) & 18.5 & 2.1 & (5) & & & \\
\hline & & & & 84 & 11.7 & -22.4 & 8.6 & (4) & & & & & & \\
\hline & & & & 76.6 & 12.8 & -24.5 & 9.7 & (10) & & & & & & \\
\hline & & & & 70.46 & 0.45 & -25.00 & 0.57 & & 21.03 & 1.46 & & 9.97 & 2.05 & \\
\hline \multirow[t]{6}{*}{$2 \mathrm{M} 0117-34$} & 19.447838 & -34.057171 & (1) & 84 & 15 & -45 & 8 & (9) & 25.56 & 0.71 & (8) & 3.258 & 1.351 & (3) \\
\hline & & & & 111.5 & 2.1 & -52.4 & 3.8 & (5) & 26.1 & 1.9 & (5) & & & \\
\hline & & & & 103.14 & 13.98 & -39.7 & 7 & (14) & & & & & & \\
\hline & & & & 102.6 & 6.9 & -42.5 & 5.6 & (10) & & & & & & \\
\hline & & & & 108.19 & 0.92 & -59.99 & 1.27 & (8) & & & & & & \\
\hline & & & & 108.54 & 0.834 & $-\mathbf{5 5 . 8 8}$ & 1.21 & & 25.63 & 0.67 & & 3.26 & 1.35 & \\
\hline \multirow{6}{*}{$2 \mathrm{M} 0045+16$} & 11.339304 & 16.579082 & (1) & 355 & 10 & -40 & 10 & (4) & 57.3 & 2 & (4) & 3.287 & 1.333 & (3) \\
\hline & & & & 354.4 & 2.2 & -51.1 & 2 & (5) & 65.9 & 1.3 & (5) & 3.29 & 0.17 & (6) \\
\hline & & & & 385 & 17 & -26 & 12 & (7) & 65.02 & 0.23 & (8) & 3.16 & 0.83 & (9) \\
\hline & & & & 374.9 & 8.5 & -27.7 & 8.4 & (10) & & & & & & \\
\hline & & & & 358.92 & 0.40 & -48.07 & 0.24 & (8) & & & & & & \\
\hline & & & & 358.82 & 0.39 & -48.08 & 0.24 & & 64.95 & 0.23 & & 3.29 & 0.17 & \\
\hline \multirow[t]{4}{*}{$2 \mathrm{M} 1551+09$} & 237.968246 & 9.687469 & (1) & -70 & 22 & -50 & 22 & (15) & 22.1 & 1.5 & (5) & -15.389 & 1.451 & (3) \\
\hline & & & & -62.1 & 0.6 & -57.7 & 0.6 & (5) & & & & & & \\
\hline & & & & -69.4 & 11.1 & -55.9 & 11.4 & (10) & & & & & & \\
\hline & & & & -62.09 & 0.59 & -57.69 & 0.60 & & 22.10 & 1.50 & & -15.39 & 1.45 & \\
\hline \multirow[t]{5}{*}{$2 \mathrm{M} 1615+49$} & 243.927302 & 49.889214 & (1) & -80 & 12 & 18 & 12 & (9) & 32.0 & 1.0 & (5) & -24.018 & 1.697 & (3) \\
\hline & & & & -23 & 34 & 41.8 & 45.5 & (16) & & & & -25.59 & 3.18 & (9) \\
\hline & & & & -92.8 & 1.2 & 15.2 & 1.8 & (5) & & & & & & \\
\hline & & & & -78.8 & 15.6 & 19.4 & 9.9 & (10) & & & & & & \\
\hline & & & & -92.51 & 1.19 & 15.43 & 1.75 & & 32.00 & 1.00 & & -24.37 & 1.50 & \\
\hline \multirow[t]{4}{*}{ 2M2154-10 } & 328.643928 & -10.925234 & (1) & 175 & 12 & 9 & 12 & (9) & 32.6 & 1.0 & (5) & -21.361 & 1.715 & (3) \\
\hline & & & & 166.8 & 1.7 & 2.2 & 2.2 & (5) & & & & & & \\
\hline & & & & 169.2 & 8.6 & -1.6 & 8.8 & (17) & & & & & & \\
\hline & & & & 167.04 & 1.65 & 2.19 & 2.10 & & 32.60 & 1.00 & & -21.36 & 1.72 & \\
\hline
\end{tabular}


Table 3

(Continued)

\begin{tabular}{|c|c|c|c|c|c|c|c|c|c|c|c|c|c|c|}
\hline Name & $\begin{array}{l}\alpha \\
\left(^{\circ}\right)\end{array}$ & $\begin{array}{c}\delta \\
\left(^{\circ}\right)\end{array}$ & Reference & $\begin{array}{c}\mu_{\alpha *} \\
\left({\left.\operatorname{mas~} \mathrm{yr}^{-1}\right)}^{-1}\right)\end{array}$ & $\begin{array}{c}\sigma_{\mu_{\alpha *}} \\
\left({\left.\operatorname{mas~} \mathrm{yr}^{-1}\right)}\right)\end{array}$ & $\begin{array}{c}\mu_{\delta} \\
\left(\operatorname{mas~yr}^{-1}\right)\end{array}$ & $\begin{array}{c}\sigma_{\mu_{\delta}} \\
\left(\operatorname{mas~yr}^{-1}\right)\end{array}$ & Reference & $\begin{array}{c}\pi \\
\text { (mas) }\end{array}$ & $\begin{array}{c}\sigma_{\pi} \\
(\mathrm{mas})\end{array}$ & Reference & $\begin{array}{c}\mathrm{RV} \\
\left(\mathrm{km} \mathrm{s}^{-1}\right)\end{array}$ & $\begin{array}{c}\sigma_{\mathrm{RV}} \\
\left(\mathrm{km} \mathrm{s}^{-1}\right)\end{array}$ & Reference \\
\hline \multirow[t]{5}{*}{ W0047+68 } & 11.751611 & 68.065102 & (11) & 387 & 4 & -197 & 4 & (12) & 82 & 3 & (12) & -17.094 & 2.732 & (3) \\
\hline & & & & 370 & 10 & -210 & 10 & (13) & 82.3 & 1.8 & (5) & -20.0 & 1.4 & (12) \\
\hline & & & & 380.7 & 1.1 & -204.2 & 1.4 & (5) & & & & & & \\
\hline & & & & 375.3 & 2.9 & -212.8 & 9.3 & (18) & & & & & & \\
\hline & & & & 380.35 & 0.99 & -203.71 & 1.30 & & 82.22 & 1.54 & & -19.40 & 1.25 & \\
\hline
\end{tabular}

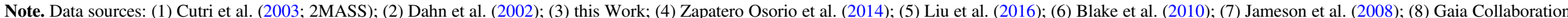

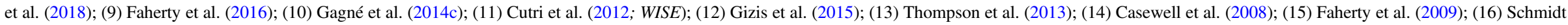
et al. (2010); (17) Gagné et al. (2014a); (18) Gizis et al. (2012); and (19) Shkolnik et al. (2017). Values in bold are weighted means. 
Table 4

Spatial/Kinematic Properties

\begin{tabular}{|c|c|c|c|c|c|c|c|}
\hline $\begin{array}{l}\text { Object } \\
\text { Name }\end{array}$ & $\begin{array}{l}\text { Sp. Type } \\
\text { (Optical) }\end{array}$ & $\begin{array}{c}X \\
(\mathrm{pc})\end{array}$ & $\begin{array}{c}Y \\
(\mathrm{pc})\end{array}$ & $\begin{array}{c}Z \\
(\mathrm{pc})\end{array}$ & $\begin{array}{c}U \\
\left(\mathrm{~km} \mathrm{~s}^{-1}\right)\end{array}$ & $\begin{array}{c}V \\
\left(\mathrm{~km} \mathrm{~s}^{-1}\right)\end{array}$ & $\begin{array}{c}W \\
\left(\mathrm{~km} \mathrm{~s}^{-1}\right)\end{array}$ \\
\hline $2 \mathrm{M} 0253+32$ & $\mathrm{M} 7 \beta$ & $-39.53 \pm 0.35$ & $21.80 \pm 0.19$ & $-20.07 \pm 0.18$ & $16.76 \pm 1.21$ & $-44.08 \pm 0.71$ & $5.54 \pm 0.62$ \\
\hline $2 \mathrm{M} 1935-28$ & M9 $\beta$ & $53.14 \pm 1.41$ & $10.05 \pm 0.27$ & $-21.54 \pm 0.57$ & $-8.89 \pm 1.10$ & $-15.47 \pm 0.45$ & $-9.11 \pm 0.56$ \\
\hline $2 \mathrm{M} 0027+05$ & M9.5 $\beta$ & $-18.30 \pm 1.20$ & $45.42 \pm 2.97$ & $-76.27 \pm 4.99$ & $-5.34 \pm 0.43$ & $0.77 \pm 0.80$ & $-6.33 \pm 1.30$ \\
\hline $2 \mathrm{M} 0241-03$ & $\mathrm{~L} 0 \gamma$ & $-27.54 \pm 1.94$ & $2.10 \pm 0.15$ & $-38.98 \pm 2.75$ & $-12.87 \pm 1.29$ & $-14.32 \pm 1.05$ & $-3.90 \pm 1.70$ \\
\hline $2 \mathrm{M} 0117-34$ & $\mathrm{~L} 1 \beta$ & $-0.50 \pm 0.01$ & $-6.05 \pm 0.16$ & $-38.58 \pm 1.01$ & $-10.09 \pm 0.32$ & $-20.48 \pm 0.60$ & $0.05 \pm 1.34$ \\
\hline $2 \mathrm{M} 0045+16$ & $\mathrm{~L} 2 \gamma$ & $-5.45 \pm 0.02$ & $9.14 \pm 0.03$ & $-11.13 \pm 0.04$ & $-21.91 \pm 0.10$ & $-14.13 \pm 0.11$ & $-5.42 \pm 0.12$ \\
\hline $2 \mathrm{M} 2154-10$ & $\mathrm{~L} 4 \beta$ & $15.10 \pm 0.47$ & $15.35 \pm 0.47$ & $-21.88 \pm 0.67$ & $-29.46 \pm 1.05$ & $-13.39 \pm 0.90$ & $0.24 \pm 1.32$ \\
\hline $2 \mathrm{M} 1551+09$ & $\mathrm{~L} 4 \gamma$ & $30.79 \pm 2.12$ & $10.87 \pm 0.75$ & $31.64 \pm 2.18$ & $-8.46 \pm 1.00$ & $-21.36 \pm 1.27$ & $-6.55 \pm 1.05$ \\
\hline $2 \mathrm{M} 1615+49$ & $\mathrm{~L} 4 \gamma$ & $4.72 \pm 0.15$ & $21.55 \pm 0.68$ & $22.18 \pm 0.70$ & $-10.40 \pm 0.40$ & $-24.77 \pm 1.07$ & $-8.09 \pm 1.11$ \\
\hline W0047+68 & $\mathrm{L} 7 \gamma$ & $-6.51 \pm 0.12$ & $10.22 \pm 0.19$ & $1.10 \pm 0.02$ & $-8.53 \pm 0.76$ & $-27.04 \pm 1.07$ & $-13.83 \pm 0.26$ \\
\hline
\end{tabular}

It is possible to handle incomplete kinematic data. Indeed, one object in this study lacks a parallax measurement. If converting to $U V W X Y Z$, a range of reasonable parallax values can be tested to see if any are consistent with moving group membership. If converting to observables, it is possible to simply not run a comparison against the predicted parallax value. This ensures that we can still evaluate kinematic memberships, though at the cost of reduced membership certainty. For a more complete discussion of the dependence of membership probabilities on observed data, see Riedel et al. (2017).

Positions for our targets come from the 2MASS (Cutri et al. 2003) catalog, with the sole exception of W0047+68 from Wide-field Infrared Survey Explorer (WISE; Cutri et al. 2012). Proper motions were sourced from a variety of papers, principally Gagné et al. (2014c), Faherty et al. (2016), Gaia Collaboration et al. (2018), Casewell et al. (2008), and other papers listed in Table 3. All of our targets have more than one proper motion measurement. Most of the proper motions are relative measurements from catalog surveys or parallax programs and are consistent at the $1 \sigma$ level for a given object, though all are generally in agreement to within \pm 10 mas $\mathrm{yr}^{-1}$, with the exception of $2 \mathrm{M} 0241-03$, one uncertain measurement of 2M0117-34, and one extremely uncertain measurement of $2 \mathrm{M} 1615+49$. We list them all individually in Table 3.

We use published parallax measurements from Faherty et al. (2016), Dieterich et al. (2014), Zapatero Osorio et al. (2014), Dahn et al. (2002), Liu et al. (2016), Gizis et al. (2015), and Gaia Collaboration et al. (2018) to obtain complete space motions (Table 3, Figures 4 and 5) and more confident group membership probabilities. Seven objects have multiple parallax measurements, which are often discrepant from each other by more than $1 \sigma$. In the case of $2 \mathrm{M} 0241-03$, the three parallaxes are only consistent at the $2 \sigma$ level, and in the case of $2 \mathrm{M} 0045$ +16 , the Gaia Collaboration et al. (2018) parallax is consistent with the Liu et al. (2016) parallax but not the Zapatero Osorio et al. (2014) parallax. In the case of $2 \mathrm{M} 0253+32$, the Faherty et al. (2016) parallax implies a larger distance than Liu et al. (2016) or Gaia Collaboration et al. (2018), though neither distance makes the target a more likely member of any known NYMG. 2M1935-28 is the only case where the Gaia Collaboration et al. (2018) and Liu et al. (2016) parallaxes do not agree even at the $2 \sigma$ level, though both distances independently make the brown dwarf a $\beta$ Pic member. With $2 \mathrm{M} 0027+05$, the Liu et al. (2016) parallax disagrees with Dahn et al. (2002), though neither parallax produces a likelihood of membership in any known NYMG.
Radial velocities have already been published for $2 \mathrm{M} 0045$ +16 (Blake et al. 2010; Faherty et al. 2016), W0047+68 (Gizis et al. 2015), 2M0241-03 (Faherty et al. 2016), 2M1615+49 (Faherty et al. 2016), and 2M1935-28 (Shkolnik et al. 2017). Our result for $2 \mathrm{M} 0045+16$ is consistent with those measured by Blake et al. (2010) and Faherty et al. (2016) well within the $1 \sigma$ uncertainties, even of the most precise measurement $\left( \pm 0.17 \mathrm{~km} \mathrm{~s}^{-1}\right)$. Our result for W0047+68 is consistent with the previous measurement to within $\sim 1 \sigma$ of our lowerprecision measurement. Both of the Faherty et al. (2016) measurements were relatively low precision $\left( \pm 3-8 \mathrm{~km} \mathrm{~s}^{-1}\right)$, upon which our measurements improve by about a factor of two or more, and our results are consistent to within $1 \sigma$ uncertainties.

All of the assembled measurements were combined with standard weighted means and weighted standard deviations. All individual results, and the weighted values (shown in bold) actually used in membership probability analysis, are shown in Table 3. UVWXYZ velocities for the objects with full kinematic information are given in Table 4.

\subsection{Membership Probabilities}

There are a number of differing approaches to kinematic moving group identification, and following Faherty et al. (2016), we consider the results of five different codes (the four used in Faherty et al. 2016 for comparison purposes, plus the newer BANYAN $\Sigma$ code) to provide a consensus approach. Four of the codes used here (BANYAN I, BANYAN II, BANYAN $\Sigma$, and LACEwING; see descriptions and references below), test against different properties: proper motion, RV, parallax, and space position. The fifth code, the convergence code from Rodriguez et al. (2013), considers only a single test for proper motion, but predicts the distance and RV. If data do not exist or are not present, the test is simply not run and the codes produce results based only on the other tests.

The convergence code presented in Rodriguez et al. (2013) exploits the fact that if all of the stars in a moving group exhibit parallel space motions, their proper motion vectors should converge at a point in space (a convergent point, analogous to the vanishing point) because of perspective effects. The code computes probabilities of membership in six moving groups (TW Hya, $\beta$ Pic, Tuc-Hor, Columba, Carina-Near, and $\mathrm{AB}$ Dor) by comparing the proper motion vector defined by $\left(\mu_{\alpha *}, \mu_{\delta}\right)$ to one pointing toward the convergent point of a given moving group. From there it predicts the associated RV and distance of the object, which can be compared to any actual measurements. 

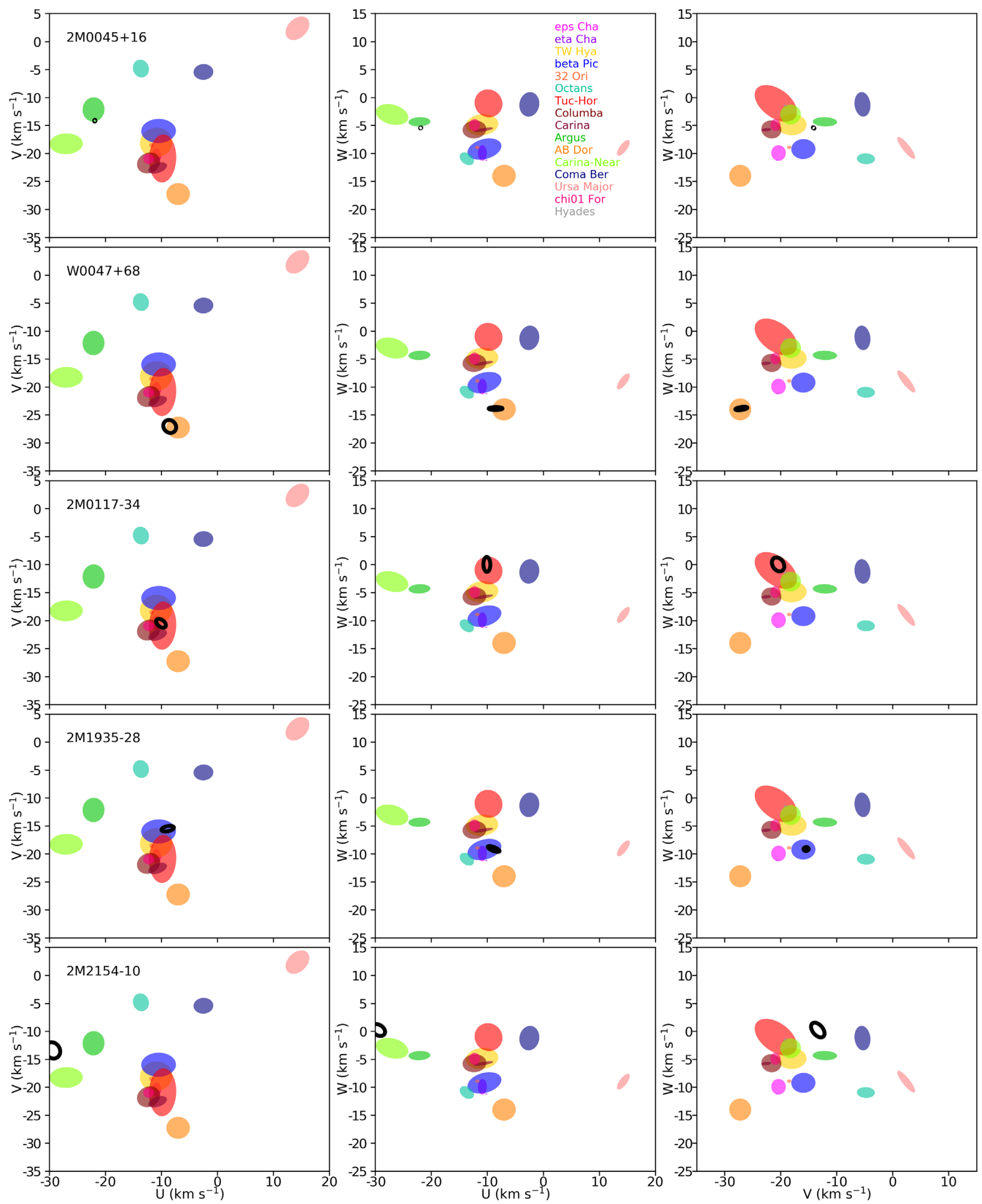

Figure 4. Projected $U V W$ space motions in the $U V, U W$, and $V W$ planes for the objects from our sample with apparent memberships in a known NYMG. The black ellipse denotes the $U V W$ phase-space position of the object relative to the known NYMGs and nearby open clusters (taken from Riedel et al. 2017), which are shown with $1 \sigma$ extents in different colors. 

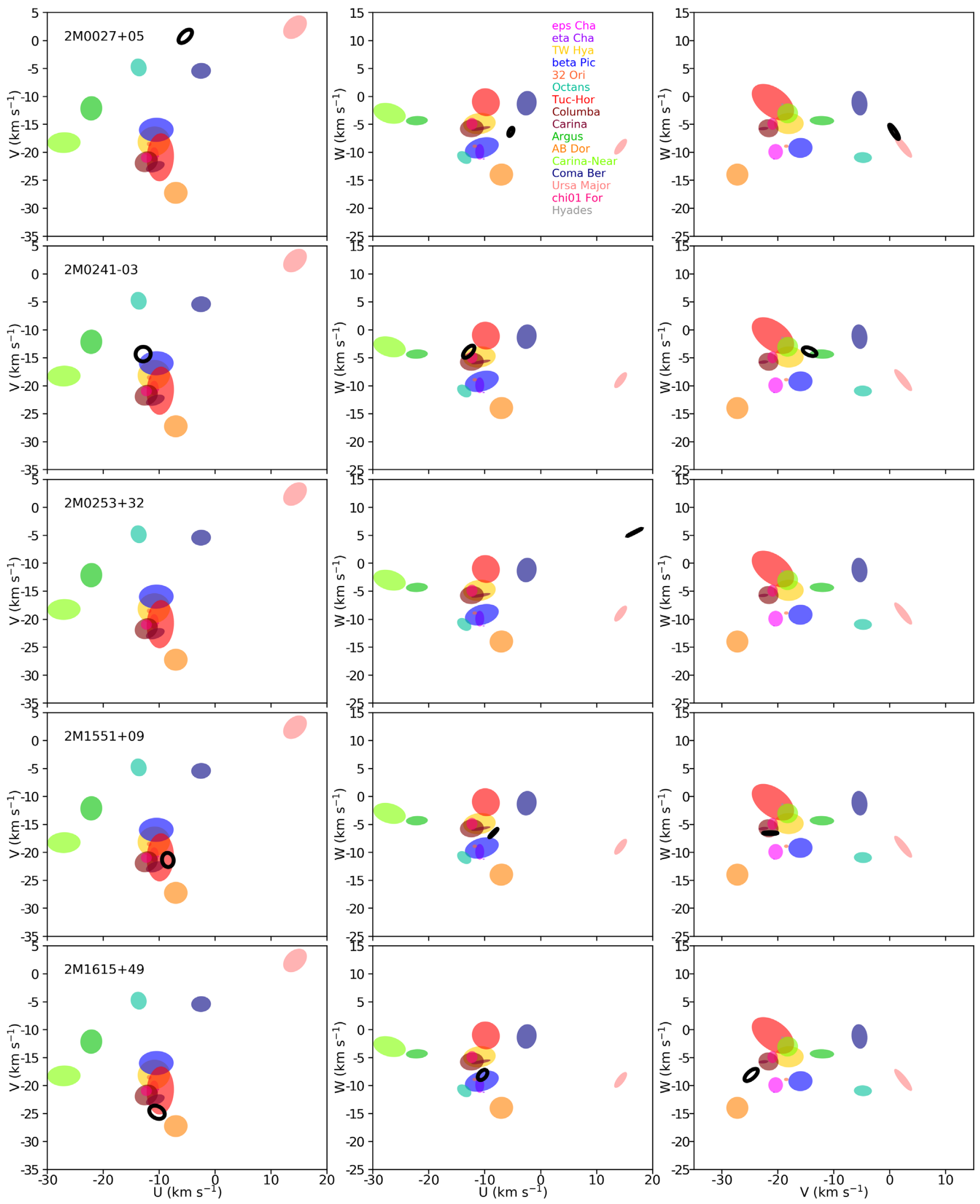

Figure 5. Same as Figure 4, showing the five objects from our sample with parallaxes but no apparent membership in a known NYMG. The $V$ velocity of $2 \mathrm{M} 0253+32$ is $-44.17 \mathrm{~km} \mathrm{~s}^{-1}$, which is outside the range of our plots. 
BANYAN I (Malo et al. 2013) uses a Bayesian formalism to evaluate which of the seven NYMGs (TW Hya, $\beta$ Pic, TucHor, Columba, Carina, Argus, and AB Dor) or a field population of which an object is most likely to be a member. It converts observables to Cartesian space. Unlike the convergence code, RV and parallax measurements are incorporated into the probability distribution rather than predicted according to possible group membership.

BANYAN II (Gagné et al. 2014c) is a modification of BANYAN I. It considers the same seven moving groups as BANYAN I, but it is based on a different set of bonafide members, assumes an uneven distribution of the number of stars in each group, and allows freely oriented moving groups in space. It takes a hybrid approach, constraining observables based on Cartesian space.

LACEwING (Riedel et al. 2017) predicts memberships in 16 NYMGs and open clusters within $100 \mathrm{pc}$ : compared to the BANYAN codes, it adds $\epsilon$ Chamæleon, $\eta$ Chamæleon, 32 Orionis, Octans, Carina-Near, Coma Berenices, Ursa Major, $\chi^{01}$ Fornax, and the Hyades. Like BANYAN II, all groups are represented as freely oriented ellipsoids with numerically proportionate populations. Unlike BANYAN II, it does not use Bayesian priors; instead it relies on the parameterized results of a large simulation of stars to translate goodness-of-fit values into membership probabilities. It operates in observational space.

BANYAN $\Sigma$ (Gagné et al. 2018) is a more refined version of BANYAN II using multivariate Gaussian models (instead of the orthogonal axis models of BANYAN II and LACEwING) which predicts memberships in 27 young moving groups and associations with ages up to $800 \mathrm{Myr}$ and distances up to $150 \mathrm{pc}$, including all of the groups in LACEwING, plus 118 Tau, Corona Australis, Upper Corona Australis, IC 2391, IC 2602, Lower Centaurus Crux, Upper Centaurus Lupus, Upper Sco, $\rho$ Oph, the Pleiades, Taurus, Platais 8, VolansCarina, and the new formulation of Argus identified in Zuckerman (2019). Like BANYAN II, it constrains the observables based on Cartesian space.

\section{Membership Results and Notes on Individual Objects}

We identify 5 of our 11 sample objects as high probability members of known NYMGs. Four of these are reconfirmations of possible memberships presented in Gizis et al. (2015), Faherty et al. (2016), Liu et al. (2016), or Shkolnik et al. (2017), and one is a new membership. The remaining six objects are found to have no membership in a known NYMG.

$2 M 0045+16$ - (L2 $\beta$ ) was identified by Gagné et al. (2014b) as a member of the roughly $50 \mathrm{Myr}$ old Argus association, using more or less the original definition of Argus from Torres et al. (2008) and by Liu et al. (2016) using BANYAN II. That identification is reconfirmed here with $90 \%-100 \%$ probabilities, which maintains this object as one of the few brown dwarfs in Argus, with an estimated mass of $25.0 \pm 4.6 M_{\text {jup }}$ (Faherty et al. 2016). Given that Argus has been kinematically (Torres et al. 2008) and chemically (De Silva et al. 2013) associated with the nearby IC 2391 open cluster, we can draw on the properties of hundreds of higher mass stars to understand this and other similarly young brown dwarfs. $2 \mathrm{M} 0045+16$ is a member of Argus, both in its original formulation (Torres et al. 2008 and subsequent, used in BANYAN I, BANYAN II, and LACEwING), which was disputed by Bell et al. (2015), and the new definition from Zuckerman (2019; used in BANYAN $\Sigma$ ).

There are three published parallaxes for $2 \mathrm{M} 0045+16$, two of which agree with each other, while a third value from Zapatero Osorio et al. (2014) is inconsistent at a $2 \sigma$ level (see Table 3). Even using discrepant parallax, we find Argus to be the most likely NYMG membership by all methods that consider membership in Argus.

As an alternative hypothesis, LACEwING suggests that 2M0045+16's kinematics are also consistent with $\beta$ Pictoris. This would make $2 \mathrm{M} 0045+16$ a significantly younger brown dwarf of roughly $25 \mathrm{Myr}$ (Mamajek \& Bell 2014) rather than $50 \mathrm{Myr}$.

We propose the 2MASS J0045+16 be considered an RV standard given the precision and stability of measurements from three different studies and its relative brightness among early L dwarfs. Its $J=13.06$ mag makes it the sixth brightest L2 and in the top 25 brightest early $(<\mathrm{L} 5) \mathrm{L}$ dwarfs, just 0.6 mag fainter than the brightest known $\mathrm{L} 2$ and $\sim 1$ mag fainter than the brightest single early L dwarf. RV measurements have previously been reported by Blake et al. (2010) from 2003 $K$-band observations $\left(3.29 \pm 0.17 \mathrm{~km} \mathrm{~s}^{-1}\right)$ and Faherty et al. (2016) from $2008 H$-band observations $\left(3.16 \pm 0.83 \mathrm{~km} \mathrm{~s}^{-1}\right)$, both also from NIRSPEC. Given the consistency of these and our measurement of $3.29 \pm 1.33$ from 2014 NIRSPEC $J$-band observations, it seems that 2MASS J0045+16 is RV stable and an optimal late-type spectral standard.

$W 0047+68-(\mathrm{L} 7 \gamma)$ has previously been identified as an AB Doradus member by Gizis et al. (2015) and Liu et al. (2016) using full $U V W X Y Z$ space motion and position fitting. We reconfirm that membership: W0047+68 is a member of AB Doradus according to every code, despite a $2 \sigma$ disagreement between our RV and that of Gizis et al. (2015). This L7 $\gamma$ object is one of the least massive known free-floating extrasolar objects, with an estimated mass of $11.8 \pm 2.6 M_{\text {Jup }}$ (Faherty et al. 2016), despite being substantially older than other brown dwarfs with a $\gamma$ gravity classification.

$2 \mathrm{M} 0117-34-(\mathrm{L} 1 \beta)$ is confirmed with our RV measurement and the parallax from Gaia Collaboration et al. (2018) as a member of Tucana-Horologium, an identification made by Faherty et al. (2016) solely on the basis of its proper motion and by Liu et al. (2016) on the basis of its proper motion and parallax. The new membership is agreed upon by every moving group code and implies that the brown dwarf is $16.4 \pm$ 3.7 $M_{\text {Jup }}$ (Faherty et al. 2016). The alternative proper motions from Casewell et al. (2008), Gagné et al. (2014c), and Liu et al. (2016) have much larger motion along the $\alpha$-axis than the proper motion calculated by Faherty et al. (2016), but all the membership codes still find membership in TucanaHorologium.

2 M1935-28-(M9 $\gamma$ ) is a member of $\beta$ Pictoris, first identified as such by Shkolnik et al. (2017). With LACEwING, BANYAN I, and BANYAN $\Sigma$, it is a moderate or high probability member; with BANYAN II, it is a lower likelihood and with the Convergence method, it is either a $\beta$ Pictoris or Columba member (see Table 5). We consider this system as a high probability member of $\beta$ Pictoris.

$2 M 2154-10-(\mathrm{L} 4 \beta)$ is identified by LACEwING, the convergence code, and BANYAN $\Sigma$ as a moderate probability member of Carina-Near, a 200-Myr-old group identified by Zuckerman et al. (2006). This makes 2M2154-10 the oldest confirmed NYMG member in the sample. Gagné et al. (2014c) 
Table 5

Membership Results

\begin{tabular}{|c|c|c|c|c|c|c|c|}
\hline $\begin{array}{l}\text { Object } \\
\text { Name }\end{array}$ & $\begin{array}{l}\text { Sp. Type } \\
\text { (Optical) }\end{array}$ & $\begin{array}{c}\text { Final } \\
\text { Membership }\end{array}$ & LACEwING & BANYAN I & BANYAN II & CONVERGE $^{\mathrm{a}}$ & BANYAN $\Sigma$ \\
\hline $2 \mathrm{M} 0253+32$ & M7 $\beta$ & None & None & Field & Field & $(\beta \text { Pic-92 })^{\mathrm{a}}$ & Field \\
\hline 2M0534-06 & M8 $\gamma$ & None & None & Field & Argus-94 & (AB Dor-100) & Field \\
\hline $2 \mathrm{M} 0027+05$ & M9.5 $\beta$ & None & None & Field & Field & (Car-Near-100) & Field \\
\hline $2 \mathrm{M} 0241-03$ & $\mathrm{~L} 0 \gamma$ & None & Field & Field & Field & Field & Field \\
\hline $2 \mathrm{M} 0117-34$ & $\mathrm{~L} 1 \beta$ & Tuc-Hor & Tuc-Hor-96 & Tuc-Hor-100 & Tuc-Hor-100 & Tuc-Hor-99 & Tuc-Hor-100 \\
\hline $2 \mathrm{M} 1615+49$ & $\mathrm{~L} 4 \gamma$ & None & AB Dor-25 & Field & (AB Dor-37) & (Tuc-Hor-86) & Field \\
\hline $2 \mathrm{M} 2154-10$ & $\mathrm{~L} 4 \beta$ & Carina-Near & Carina-Near-53 & Field $^{b}$ & Field & Field & Car-Near-89 \\
\hline W0047+68 & $\mathrm{L} 7 \gamma$ & AB Dor & AB Dor-100 & AB Dor-100 & AB Dor-100 & AB Dor-85 & AB Dor-100 \\
\hline
\end{tabular}

Notes. The quoted membership probability is the highest membership probability for the most commonly identified moving group, considering every permutation of kinematic data. Probabilities in parentheses are below the quality threshold: LACEwING: $20 \%$; BANYAN codes, $90 \%$; convergence code, $80 \%$. See the discussion in Section 3.3 .

${ }^{\text {a }}$ Values in parentheses are inconsistent with actual membership. Either the probability is too low for that particular code, or (particularly for the convergence code) the predicted distance, space position, or RVs are inconsistent with membership or actual measurements.

${ }^{b}$ The convergence code and BANYAN $\Sigma$ do not consider membership in Argus. Only LACEwING, the convergence code, and BANYAN $\Sigma$ consider membership in Carina-Near.

found 2M2154-10 to be a member of Argus, which we do not reproduce due to a disagreement in the RV: as a member of Argus it should have an RV of roughly $-14 \mathrm{~km} \mathrm{~s}^{-1}$, while we measure an $\mathrm{RV}$ of $-21 \pm 2 \mathrm{~km} \mathrm{~s}^{-1}$.

The remaining targets-2M0027+05 $(\mathrm{M} 9.5 \beta), 2 \mathrm{M} 0253+32$ (M7 $\beta$ ), 2M0534-06 (M8 $\gamma$ ), 2M0241-03 (L0 $\gamma), 2 \mathrm{M} 1551+09$ $(\mathrm{L} 4 \gamma)$, and $2 \mathrm{M} 1615+49(\mathrm{~L} 4 \gamma)$ were all identified having ambiguous NYMG membership by Faherty et al. (2016) and are not found to be likely members of any known NYMG with the addition of our RV measurements and Gaia Collaboration et al. (2018) astrometry.

The convergence method predicts that $2 \mathrm{M} 0253+32$ is a member of $\beta$ Pic with a predicted RV of $5.5 \mathrm{~km} \mathrm{~s}^{-1}$, but our measured $\mathrm{RV}$ is $-34.5 \mathrm{~km} \mathrm{~s}^{-1}$. We therefore conclude that $2 \mathrm{M} 0253+32$ is not a $\beta$ Pic member.

$2 \mathrm{M} 1615+49$ appears to be a rapid rotator (see Figure 10) but does not otherwise distinguish itself. LACEwING finds it to be a potential member of $\mathrm{AB}$ Doradus, though at low probability; the convergence code finds it a possible member of Tuc-Hor (with a predicted RV of $-15 \mathrm{~km} \mathrm{~s}^{-1}$, which does not match our measured $-24 \mathrm{~km} \mathrm{~s}^{-1}$ ), and BANYAN I, BANYAN II, and BANYAN $\Sigma$ find it is not a member of any group at a probability above the threshold of interest.

The expected RV for $2 \mathrm{M} 1551+09$ if it were a member of $\beta \mathrm{Pic},-17 \mathrm{~km} \mathrm{~s}^{-1}$, is consistent with the actual measured velocity of $-15 \mathrm{~km} \mathrm{~s}^{-1}$, but only the convergence code finds that membership and at a low probability. The expected distance for a $\beta$ Pic member with the proper motion of 2 M 1551 +09 would place it very far spatially from the known members of $\beta$ Pic (a condition the BANYAN codes and LACEwING consider), which means that even if its parallax-determined distance matches the expected distance of $30 \mathrm{pc}$, the object cannot be a member.

2M0241-03 has five published proper motions (Casewell et al. 2008; Gagné et al. 2014c; Zapatero Osorio et al. 2014; Faherty et al. 2016; Liu et al. 2016) and three parallaxes (Zapatero Osorio et al. 2014; Faherty et al. 2016; Liu et al. 2016), which only agree with each other at the $2 \sigma$ level. This system has been considered a member of Tucana-Horologium since Gagné et al. (2014c), but with our weighted parallax we find no such membership. Using the Faherty et al. (2016) and Zapatero Osorio et al. (2014) 14 parallaxes individually, the brown dwarf is still not a member of any moving group. Liu et al. (2016) placed it in Tuc-Hor with an $82 \%$ likelihood using BANYAN II (a lower probability than our threshold for BANYAN II) using parallax and proper motion, but with our astrometry BANYAN II gives us an $88 \%$ membership (also below the threshold) in $\beta$ Pic instead. LACEwING does reproduce membership in Tuc-Hor at a low 30\% probability, and we note that LACEwING gives a higher $(46 \%)$ chance of membership in Columba. The convergence code suggests a low probability of membership in Carina-Near if the system is at $80 \mathrm{pc}$, which it is not.

Ultimately, the reason it is not in Tuc-Hor is a combination of factors: if its (combined) proper motion and RV were to imply the best possible space velocity match to Tuc-Hor, the brown dwarf would need to be closer to $64 \mathrm{pc}$ away, which not even the Liu et al. (2016) parallax (54 pc) agrees with, while (simultaneously) being at that appropriate distance would put it approximately $40 \mathrm{pc}$ away (over $2 \sigma$ ) from the bulk of the TucHor moving group.

For now, we suspect that these objects are members of a young field population, which Riedel et al. (2017) has shown to be quite substantial.

\section{Discussion}

\subsection{RV Measurements of Very-low-mass Objects}

Typically, high-resolution spectra are cross-correlated against spectra of objects with similar spectral types, effectively doubling the required observing time, which can be on the order of several hours for intrinsically faint very-low-mass stars and brown dwarfs. For example, Prato et al. (2015) used a similar cross-correlation method to ours for measuring the RVs of very-low-mass objects, but restricted that comparison to objects with similar spectral types. In order to optimize the efficiency of RV measurements for very-low-mass objects, we 

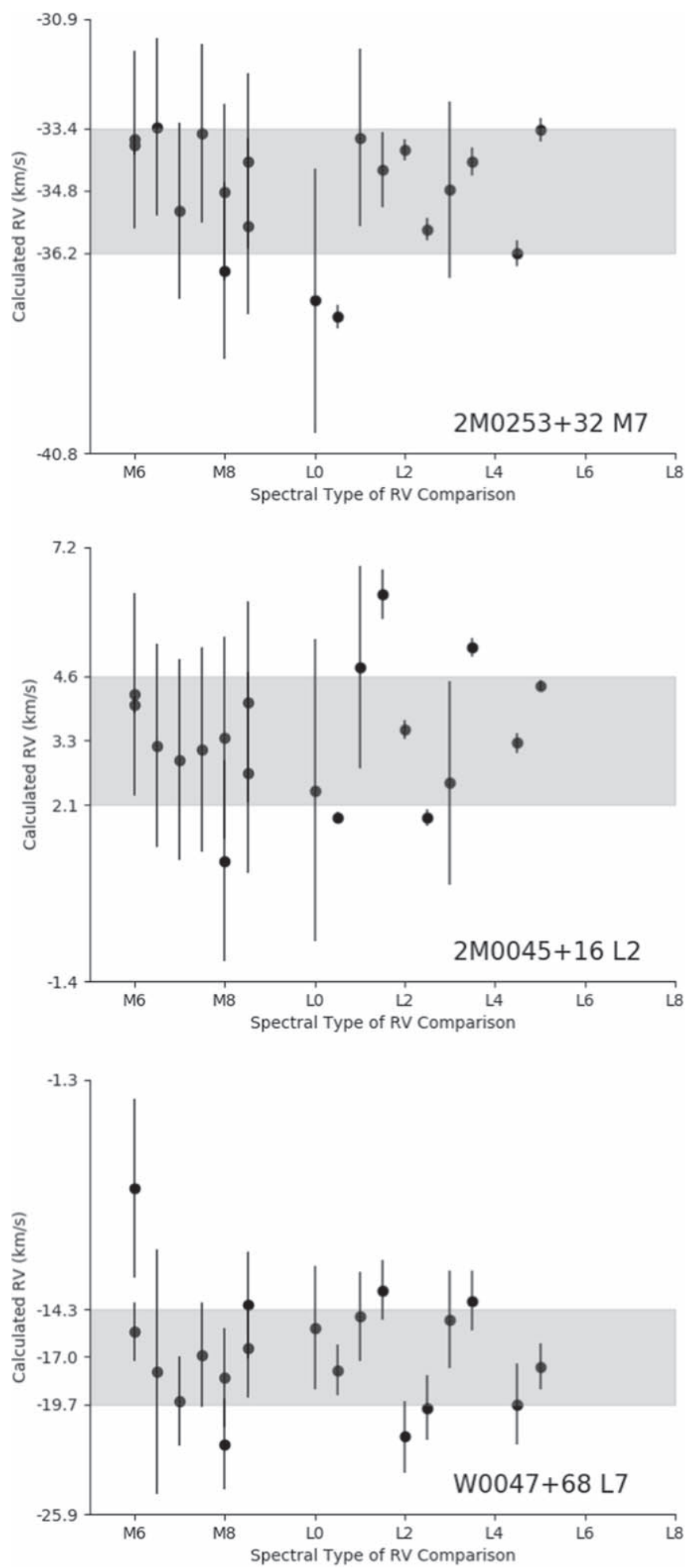

Figure 6. Measured RV ( $\mathrm{km} \mathrm{s}^{-1}$ ) vs. spectral type of each comparison object for $2 \mathrm{M} 0253+32$ (top), $2 \mathrm{M} 0045+16$ (middle), and W0047+68 (bottom). The gray bar represents the RV $1 \sigma$ uncertainty range on the final results for each target. The RV of the target is calculated by cross-correlating each target and comparison pair, and does not show any correlation to or dependence on the difference in spectral type between the comparison and target objects.

test the dependence of the precision and accuracy of RV results on the spectral type of the comparison object.

Figure 6 shows the spectral type of each RV comparison, as listed in Table 2, as a function of the calculated RV of the target object. The gray bar indicates the $1 \sigma$ uncertainty on the final RV measurement of the target, as listed in Table 3. The three panels show the objects of earliest and latest spectral type in our sample, 2M0253+32 (M7, top panel) and W0047+68 (L7, bottom panel), as well as the object of median spectral type, $2 \mathrm{M} 0045+16$ (L2, middle panel). The same test was done for all 11 objects in our sample. We see no correlation between spectral type of the comparison object and the precision or accuracy of the individual RV measurement, compared to the final value. Thus, we show that a cross-correlation comparison of objects can be as different as \pm 5 spectral types from the target object. This is similar to the findings of Newton et al. (2014) for M dwarfs (their Section 8.2).

These results can improve the efficiency of observations required for using the cross-correlation technique for measuring the RVs of late-type objects by loosening the requirements for the comparison object's spectral type similarity to that of the target. If a close spectral type match is not required, less observing time needs to be spent on assembling (from previously obtained spectra, or new observations) a library of high-resolution comparison spectra, and the expense of making RV measurements of late-type objects decreases markedly.

\subsection{NIR Colors of Young Very-low-mass Objects}

By virtue of their selection as objects with spectral indicators of youth, most of our sample has redder near- and mid-infrared colors than expected for normal L dwarfs (Figure 1), with the exception of $2 \mathrm{M} 0253+32$, which is consistent with the colors of a normal L dwarf of the same spectral type. The degree of reddening is fairly consistent across all near- and mid-infrared color combinations, with a few exceptions: in $H-K, 2 \mathrm{M} 0027$ +05 is bluer than (but consistent with) a normal L dwarf; in $K-W 1,2 \mathrm{M} 0534-06$ is likewise bluer or consistent with a normal L dwarf. Neither of these effects are due to poor precision photometry, they appear to be real (or perhaps variable) features of the objects themselves.

The NIR colors alone are not a sufficient gauge of age. The most consistently discrepant objects in the sample, which are also generally the objects with the largest color offsets from normal brown dwarfs, are 2M0241-03, 2M1615+49, 2M2154 -10 , and W0047+68; W0047+68 is identified as an AB Dor (120 Myr) member and is both the potentially oldest identified member in the sample and the coldest brown dwarf in the sample, while the $\beta$ Pictoris ( $25 \mathrm{Myr}$ ) member, $2 \mathrm{M} 1935-28$, is just above the envelope of young brown dwarfs in Figure 1.

\subsection{K I Line Strengths}

Measurements of gravity-sensitive spectral lines may provide a more reliable indicator of youth than NIR colors alone (e.g., Faherty et al. 2012). The neutral alkali metal absorption lines like those of $\mathrm{Na}$ I and K I are weaker in lower surface gravity atmospheres (e.g., Schlieder et al. 2012), which translates to smaller equivalent widths (EWs). This provides a way to test if red objects are truly young and low surface gravity, or simply red because of dustier atmospheres. With our high-resolution spectra we can measure the strength and width of gravity-sensitive lines and test this directly, which we describe below. We can also determine if these objects are rapid rotators by measuring the FWHM of the lines. 

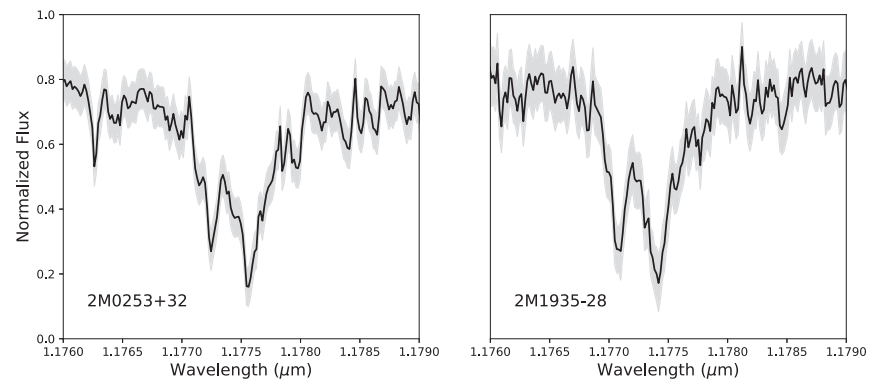

Figure 7. K I triplet for $2 \mathrm{M} 0253+32$ (left panel) and 2M1935-28 (right panel).

NIRSPEC orders $61(1.24-1.26 \mu \mathrm{m})$ and $65(1.165-1.182 \mu \mathrm{m})$ each contain a K I doublet (in order 65 the lines are sometimes resolvable into a triplet, see Figure 7) that has been shown to be sensitive to temperature (e.g., McLean et al. 2007) and surface gravity (e.g., McGovern et al. 2004; Rice et al. 2010). Therefore we measure these line strengths and compare them to those of field-age objects to evaluate additional indicators of youth for our sample. Because of the only occasionally resolved triplet in order 65 , which is also typically of lower $\mathrm{S} / \mathrm{N}$, we concentrate our analysis on the order 61 doublet.

Following the methods of Alam \& Douglas (2016), we quantify the strengths of the $\sim 1.25 \mu \mathrm{m} \mathrm{K} \mathrm{I} \mathrm{lines} \mathrm{for} \mathrm{our}$ sample by computing EWs and FWHM using the PHEW: PytHon EWs ${ }^{17}$ code. We measure EWs using a zeroth-order fit to the pseudo-continuum, defined as the average flux outside of the absorption line within a $1.241-1.246 \mu \mathrm{m}$ window, and a Voigt profile fit to the absorption line. The EW is calculated by integrating the pseudo-continuum level minus the spectrum over the selected range. Uncertainties were estimated via 1000 Monte Carlo iterations. We report the means and standard deviations of these measurements in Table 6 . Figure 8 presents an example of these measurements for the $\lambda=1.2525 \mathrm{~K}$ I line from the spectrum of $2 \mathrm{M} 0045+16$. Line strength measurements, compared with results for field objects from M. K. Alam et al. (2019, in preparation) and McLean et al. (2003), are presented in Figures 9 and 10. The complete data set for both field and suspected young objects is presented in Table 6.

These results follow the general pattern indicated by other studies of the K I lines, e.g., Allers \& Liu (2013; their Figure 23), Gagné et al. (2015c; their Figure 6), and Martin et al. (2017; their Figure 3) and indicate that our suspected young sample exhibits lower surface gravities, as expected for objects with $\beta$ and $\gamma$ gravity designations. Our results are not directly comparable to those of the aforementioned papers due to our higher spectral resolution. Those papers used moderate ( $R \sim 750-2000)$ spectra, while our $R \sim 20,000$ spectra yields more precise measurements, higher EWs for field stars, a decreased sensitivity to the $\mathrm{FeH}$ feature overlapping with the $1.2436 \mu \mathrm{m}$ line, and correspondingly more distinction between field objects and our suspected young sample, even at the extremes where Allers \& Liu (2013) could only determine an EW-based gravity classification and saw no differences between young and field stars in their $\mathrm{K} \mathrm{I}$ index.

The FWHM measurements (Figure 10) demonstrate that almost all of our targets have lower $v \sin i$ than the field objects. Two objects, 2M0241-03 (L0 $\gamma)$ and 2M1615+49 (L4 $\gamma$ ), are possibly rapid rotators and/or viewed more edge-on than the

\footnotetext{
17 https://github.com/munazzaalam/PHEW/
}

other young objects, which would broaden their gravityweakened lines, as evidenced by their higher FWHM measurements but similar EWs to the other candidate young objects.

\subsection{Consistency of Age Indicators}

Near-infrared colors may indicate possible youth, but they have never been considered sufficient to determine specific ages for young brown dwarfs, as noted in Section 5.2. Spectroscopic measurements are more useful for evaluating youth, but here too there are limitations. All of our objects have been classified as either $\beta$ or $\gamma$ gravity classes according to their red-optical spectra (Table 1), and most objects have INT-G or VL-G gravity classes on the NIR Allers \& Liu (2013) spectral system. As shown in Figure 9, all of our targets have weaker (lower EW) K I doublet lines than field-age dwarfs of comparable spectral type, indicating youth, though there is substantial overlap between $\beta$ and $\gamma$ gravity classifications. Furthermore, gravity-related spectral type suffixes themselves do not appear to track directly with age; our $50 \mathrm{Myr}$ old Argus member $2 \mathrm{M} 0045+16$ is an $\mathrm{L} 2 \beta$, while our $125 \mathrm{Myr}$ old $\mathrm{AB}$ Doradus member W0047+68 is classified as L7 $\gamma$. We therefore cannot assign even relative ages based on line strength or gravity measurements alone. We also do not consider it problematic that $\mathrm{W} 0047+68$ shows signs of youth when M-dwarf members of AB Doradus typically do not have identifiable low surface gravity features (Schlieder et al. 2012), as it is a much cooler, and therefore lower-mass object that may be evolving more slowly.

Even the non-spectrophotometric property of kinematic memberships has limitations. Taken in total, the line strengths and colors indicate that all the objects in our sample are young (if not precisely how young), but we can only connect five of them with NYMGs that confirm a young age. The failure to connect the remaining objects to an NYMG could be explained by one of four of the following possibilities.

1. The NYMG identification algorithm may be flawed because it is based on inaccurate or incomplete assumptions of how to best identify NYMGs.

2. We may have insufficiently precise kinematic data for the late-type object, or an inaccurate understanding of the parameters of the NYMG itself.

3. The object may be a member of an as-yetunknown NYMG.

4. The object may be a young unassociated or field object, for example the product of a one-off star formation event, as suggested by Riedel et al. (2017) and Gagné et al. (2018).

We can attempt to explore the first two possibilities using multiple techniques, as the five different moving group identification codes all have different algorithms and different parameterizations of the NYMGs. Even so, the codes agree that most of our targets are not members of any known NYMG, strengthening the probability of the third and/or fourth options.

The current situation is thus: we have photometric color, gravity classification, and individual line strengths (EWs and FWHM). They frequently disagree with each other about the degree to which an object is young, particularly when compared to the age implied by kinematic membership in an NYMG. There is as of yet no simple spectrophotometric indicator (or group of indicators) that reliably indicates age, so 
Table 6

Spectral Line Measurements

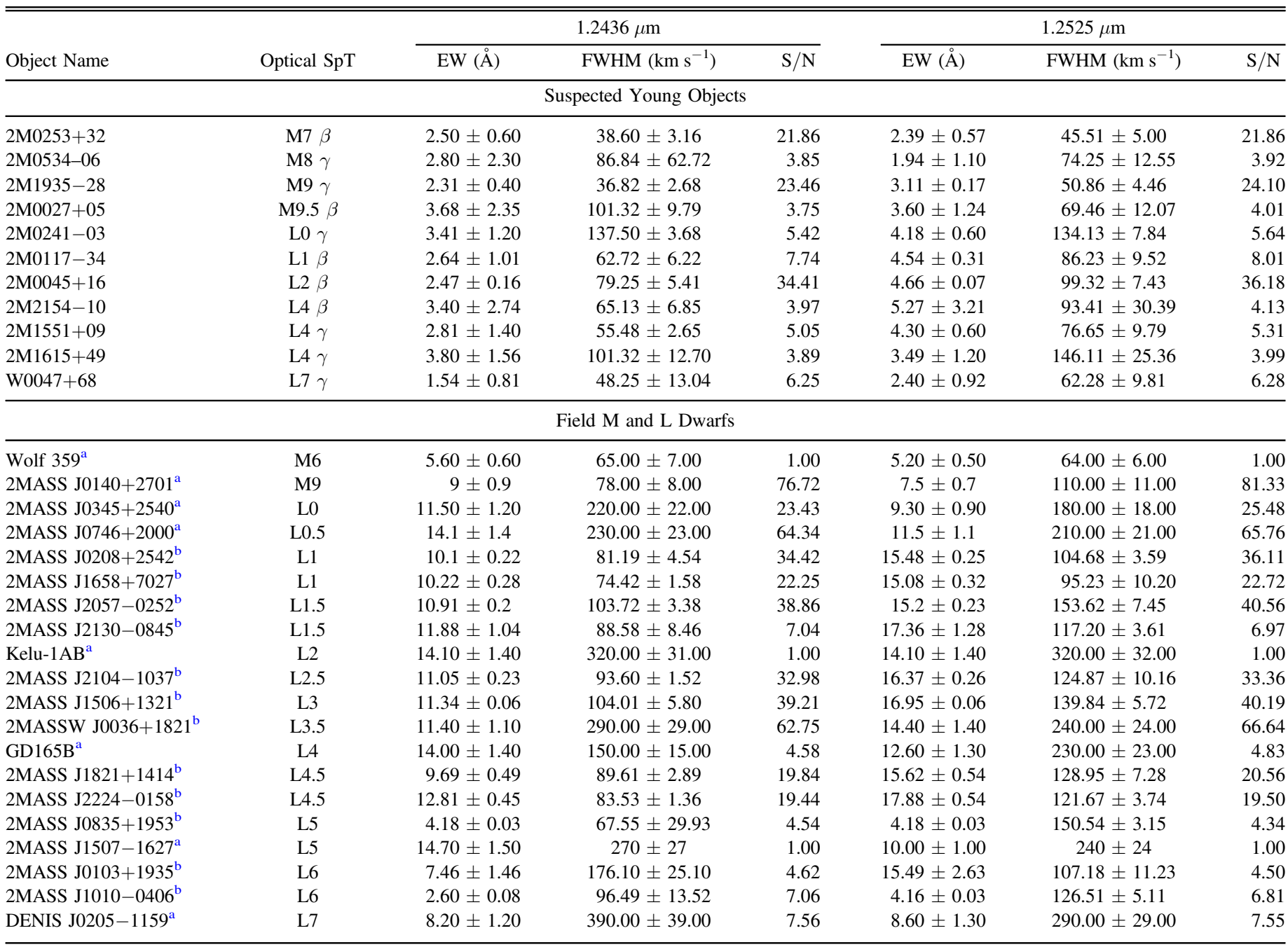

Notes. Objects are grouped by spectral type and then listed in order of R.A.

${ }^{a}$ Originally published in McLean et al. (2007).

${ }^{\mathrm{b}}$ Originally published in M. K. Alam et al. (2019, in preparation).

we are still limited to saying that by our aggregate analysis of the various metrics, the objects are likely young. Only the five objects we can connect to an NYMG can give us an age; for the other six, all we can say is that they are likely young, and there is no reason to suspect they are not as young as the confirmed NYMG members in the sample.

\section{Conclusions}

In this paper we have presented new high-resolution NIR spectroscopy of 11 red, low-gravity, late-type objects. Using new RV measurements derived from that spectroscopy, and proper motion and parallax measurements from literature sources, we reconfirm membership of four objects in NYMGs. We also identify a new member of Carina-Near and confidently rule out six objects as members of the known NYMGs. These objects remain as interesting targets for study, though we cannot currently determine their ages or origins.

Our study also adds more evidence to the hypothesis (such as proposed by Riedel et al. 2017 and Gagné et al. 2018) that there are other populations of young objects in the solar neighborhood yet to be discovered, whether they are new NYMGs or a genuinely unassociated field population of young objects. The six objects we conclusively rule out as members of the known NYMGs are an indistinguishable population, spectroscopically and photometrically, from the confirmed NYMG members. There is no reason to say that they are not young, beyond lack of group membership.

We also presented evidence that the accuracy of the crosscorrelation technique is not dependent on close spectral type matches. Previously, it was thought that spectral types of standard stars had to be as close as possible to the spectral type of the target-within two subtypes-for the cross-correlation $\mathrm{RV}$ technique. The power of this technique in face of the spectral type discrepancy is due to the strength and regularity of the $\mathrm{FeH}$ lines in cool star spectra, the rectification step where a third-order polynomial fit corresponding to the overall shape of the spectrum is removed, and the use of multiple comparison spectra in a weighted measurement. The end result is proof that collecting an extensive library of standards at every spectral type 


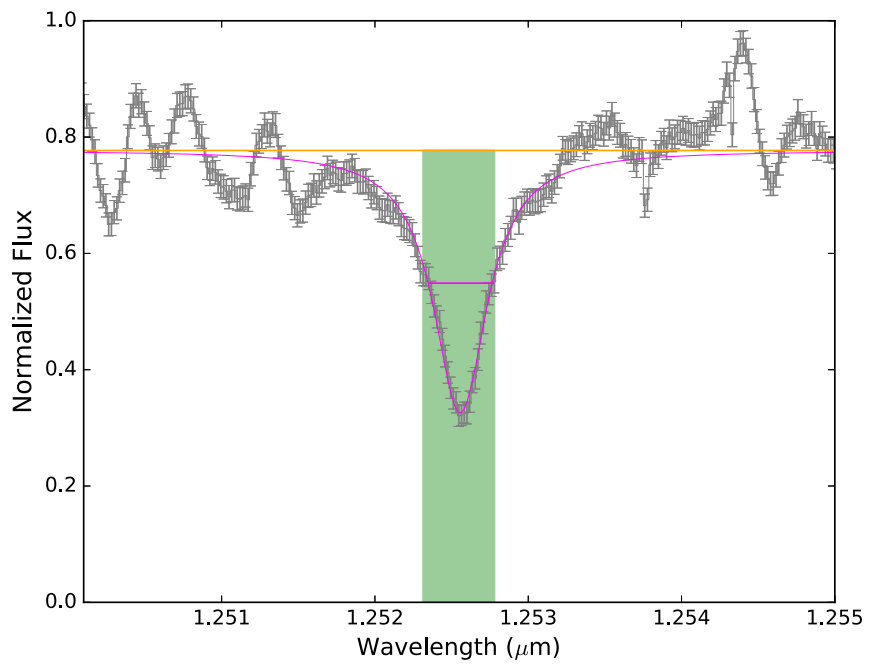

Figure 8. Subsection of NIRSPEC dispersion order 61 for $2 \mathrm{M} 0045+16$ centered on the $\lambda=1.2525 \mathrm{~K} \mathrm{I}$ line to demonstrate the line strength measurement methods of Alam \& Douglas (2016). The yellow line represents the defined pseudo-continuum, the purple curve the Voigt profile, and the purple horizontal line the FWHM. The shaded green region represents the equivalent width.
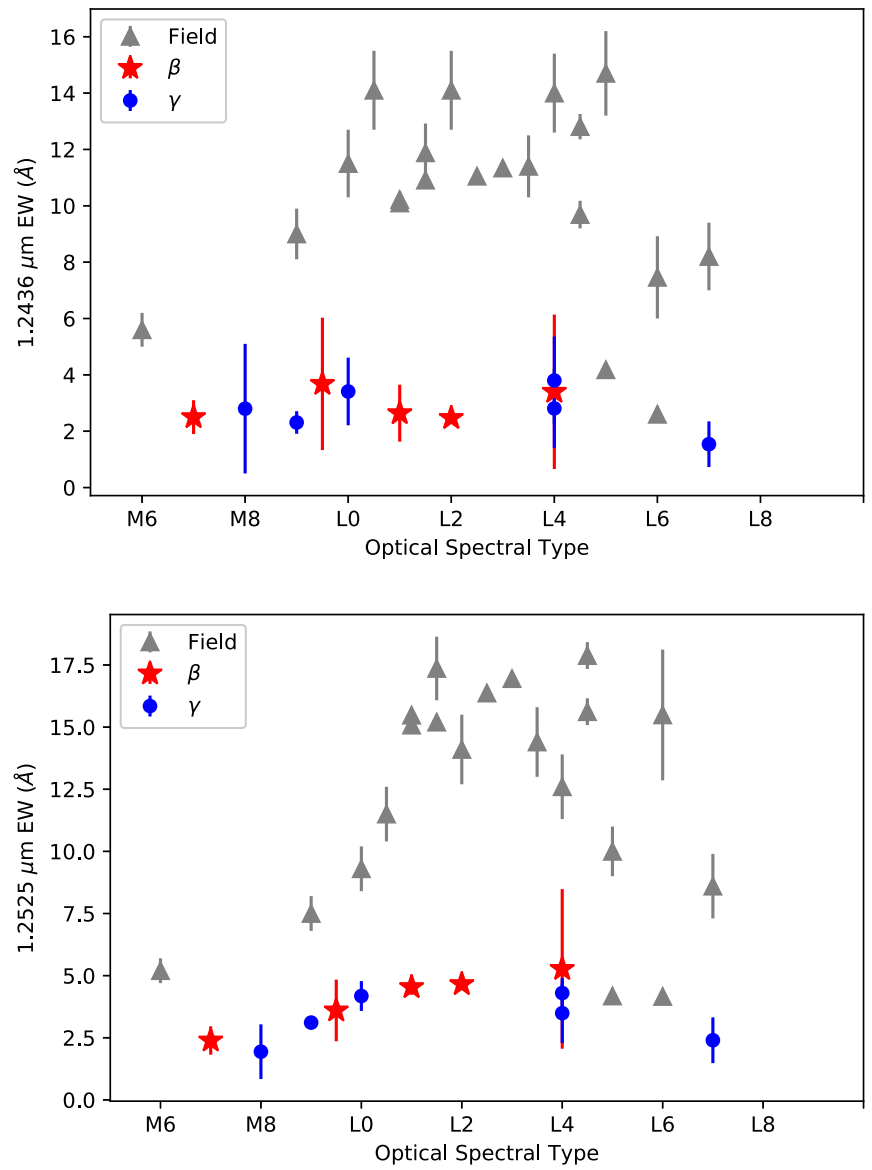

Figure 9. K I equivalent width vs. optical spectral type for our sample of candidate young, unusually red objects organized by gravity class (red stars, blue circles) compared to field objects (gray triangles) for the order 61 lines at $1.2436 \mu \mathrm{m}$ (top panel) and $1.2525 \mu \mathrm{m}$ (bottom panel) lines.

is not necessary to achieve kilometer-per-second precision RVs, and therefore shows that the technique of cross-correlation is cheaper and easier to implement than previously thought.

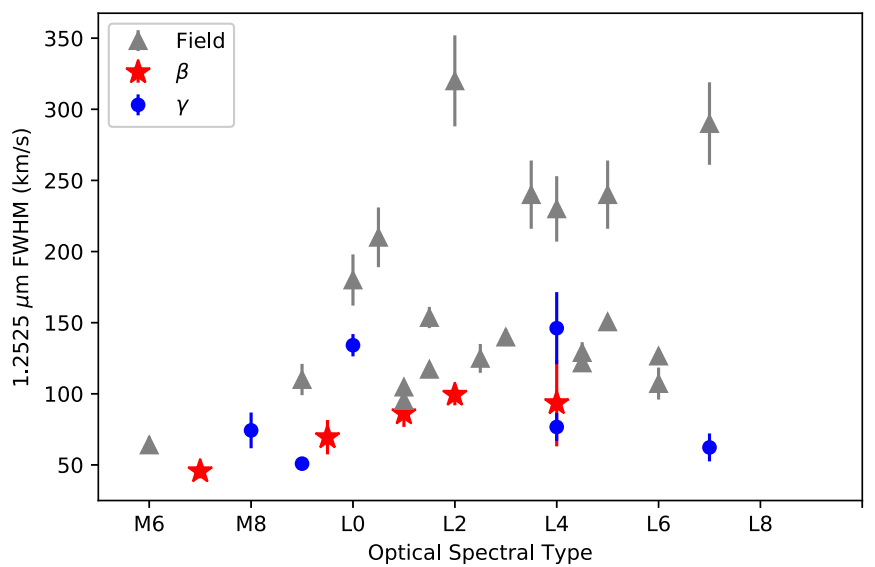

Figure 10. K I FWHM vs. optical spectral type for our sample of candidate young, unusually red objects compared to field objects for the order 61 line at $1.2525 \mu \mathrm{m}$ line. Colors are the same as Figure 9.

The authors wish to thank the staff of the Keck Observatory for their outstanding support, including Luca Rizzi, Jim Lyke, Cynthia Wilburn, Terry Stickel, Jason McIlroy, Heather Hershley, and Barbara Schaefer. Observing assistance from Kay Hiranaka was greatly appreciated. The authors are grateful for assistance with references from J.T. Wright and E.R. Newton via Twitter, and J. Gagne for help with the BANYAN II and BANYAN $\Sigma$ codes. A.R.R., V.D., and E.L.R. were responsible for writing the majority of the paper. A.R.R. was responsible for the youth analysis, conclusions, and system notes. V.D. and E.A. were responsible for the NIRSPEC data reduction and description in the paper. V.D., E.L.R., E.A., and A.R.R. were responsible for the RV analysis. M.K.A. was responsible for the line measurements, and description of PHEW. K.L.C. was responsible for the spectral typing and advising of V.D. and E.A.. J.K.F. was responsible for the general survey outline.

This material is based upon work supported by the National Science Foundation under grant Nos. AST-1313278, AST1313132, and AST-1153335. E.A. acknowledges support from the National Science Foundation Graduate Research Fellowship under grant No. DGE 1752814. This research was supported in part by NASA through the American Astronomical Society's Small Research Grant Program. This research has made use of the NASA/IPAC Infrared Science Archive, which is operated by the Jet Propulsion Laboratory, California Institute of Technology, under contract with the National Aeronautics and Space Administration. This publication makes use of data from the Two Micron All Sky Survey, which is a joint project of the University of Massachusetts and the Infrared Processing and Analysis Center, funded by the National Aeronautics and Space Administration and the National Science Foundation. This research has benefited from the M, L, and T dwarf compendium housed at DwarfArchives. org and maintained by Chris Gelino, Davy Kirkpatrick, and Adam Burgasser. This research has made use of the SIMBAD database, operated at CDS, Strasbourg, France and NASA's Astrophysics Data System. The work of A.R.R. was supported by a NASA Keck PI Data Award, administered by the NASA Exoplanet Science Institute. Keck telescope time was granted by NOAO, through the Telescope System Instrumentation Program (TSIP). TSIP is funded by NSF. Data presented herein were obtained at the W. M. Keck Observatory from telescope time allocated to the National Aeronautics and Space 
Administration through the agency's scientific partnership with the California Institute of Technology and the University of California. The Observatory was made possible by the generous financial support of the W. M. Keck Foundation. The authors wish to recognize and acknowledge the very significant cultural role and reverence that the summit of Maunakea has always had within the indigenous Hawaiian community. We are most fortunate to have the opportunity to conduct observations from this mountain.

Facility: Keck:II (NIRSPEC).

\section{ORCID iDs}

Adric R. Riedel 주 https://orcid.org/0000-0003-1645-8596 Victoria DiTomasso (1) https://orcid.org/0000-00030741-7661

Emily L. Rice (1) https://orcid.org/0000-0002-3252-5886

Munazza K. Alam (ㅈ) https://orcid.org/0000-0003-4157-832X

James Crook (1) https://orcid.org/0000-0003-1618-5540

Kelle L. Cruz iㅣ https://orcid.org/0000-0002-1821-0650

Jacqueline K. Faherty (iㅣ https://orcid.org/0000-0001-

6251-0573

\section{References}

Alam, M., \& Douglas, S. 2016, PHEW: PytHon Equivalent Widths, Zenodo, doi:10.5281/zenodo. 47889

Allers, K. N., \& Liu, M. C. 2013, ApJ, 772, 79

Barrado y Navascués, D., Stauffer, J. R., Song, I., \& Caillault, J. 1999, ApJL, 520, L123

Bell, C. P. M., Mamajek, E. E., \& Naylor, T. 2015, MNRAS, 454, 593

Beuzit, J., Feldt, M., Dohlen, K., et al. 2008, Proc. SPIE, 7014, 701418

Binks, A. S., \& Jeffries, R. D. 2014, MNRAS, 438, L11

Blake, C. H., Charbonneau, D., \& White, R. J. 2010, ApJ, 723, 684

Casewell, S. L., Jameson, R. F., \& Burleigh, M. R. 2008, MNRAS, 390, 1517

Chubak, C., Marcy, G., Fischer, D. A., et al. 2012, arXiv:1207.6212

Crepp, J. R., Principe, D. A., Wolff, S., et al. 2018, ApJ, 853, 192

Cruz, K. L., Kirkpatrick, J. D., \& Burgasser, A. J. 2009, AJ, 137, 3345

Cutri, R. M., Skrutskie, M. F., \& van Dyk, S. 2003, The IRSA 2MASS All-Sky Point Source Catalog, NASA/IPAC Infrared Science Archive, http://www. ipac.caltech.edu/2mass/releases/allsky/doc/

Cutri, R. M., Wright, E. L., \& Conrow, T. 2012, Explanatory Supplement to the WISE All-Sky Data Release Products, http://wise2.ipac.caltech.edu/ docs/release/allsky/expsup/

Dahn, C. C., Harris, H. C., Vrba, F. J., et al. 2002, AJ, 124, 1170

De Silva, G. M., D'Orazi, V., Melo, C., et al. 2013, MNRAS, 431, 1005

Dieterich, S. B., Henry, T. J., Jao, W.-C., et al. 2014, AJ, 147, 94

Dupuy, T. J., Liu, M. C., \& Ireland, M. J. 2014, ApJ, 790, 133

Dupuy, T. J., Liu, M. C., \& Leggett, S. K. 2015, ApJ, 803, 102

Faherty, J. K., Burgasser, A. J., Cruz, K. L., et al. 2009, AJ, 137, 1

Faherty, J. K., Burgasser, A. J., Walter, F. M., et al. 2012, ApJ, 752, 56

Faherty, J. K., Rice, E. L., Cruz, K. L., Mamajek, E. E., \& Núñez, A. 2013, AJ, 145,2

Faherty, J. K., Riedel, A. R., Cruz, K. L., et al. 2016, ApJS, 225, 10

Gaia Collaboration, Brown, A. G. A., Vallenari, A., et al. 2018, A\&A, 616, A1 Gagné, J., Burgasser, A. J., Faherty, J. K., et al. 2015a, ApJL, 808, L20

Gagné, J., Faherty, J. K., Cruz, K., et al. 2014a, ApJL, 785, L14
Gagné, J., Faherty, J. K., Cruz, K. L., et al. 2015b, ApJS, 219, 33

Gagné, J., Faherty, J. K., Mamajek, E. E., et al. 2017, ApJS, 228, 18

Gagné, J., Lafrenière, D., Doyon, R., et al. 2014b, ApJL, 792, L17

Gagné, J., Lafrenière, D., Doyon, R., Malo, L., \& Artigau, É. 2014c, ApJ, 783,121

Gagné, J., Lafrenière, D., Doyon, R., Malo, L., \& Artigau, É. 2015c, ApJ, 798, 73

Gagné, J., Mamajek, E. E., Malo, L., et al. 2018, ApJ, 856, 23

Gizis, J. E. 2002, ApJ, 575, 484

Gizis, J. E., Allers, K. N., Liu, M. C., et al. 2015, ApJ, 799, 203

Gizis, J. E., Faherty, J. K., Liu, M. C., et al. 2012, AJ, 144, 94

Greenbaum, A. Z., Pueyo, L., Ruffio, J.-B., et al. 2018, AJ, 155, 226

Jameson, R. F., Casewell, S. L., Bannister, N. P., et al. 2008, MNRAS, 384, 1399

Jeffries, R. D. 2012, in EAS Publications Ser. 57, Low-Mass Stars and the Transition Stars/Brown Dwarfs, ed. C. Reylé, C. Charbonnel, \& M. Schultheis (Les Ulis: EDP Sciences), 45

Kirkpatrick, J. D., Barman, T. S., Burgasser, A. J., et al. 2006, ApJ, 639, 1120

Konopacky, Q. M., Ghez, A. M., Barman, T. S., et al. 2010, ApJ, 711, 1087

Kraus, A. L., Shkolnik, E. L., Allers, K. N., \& Liu, M. C. 2014, AJ, 147, 146

Lew, B. W. P., Apai, D., Zhou, Y., et al. 2016, ApJL, 829, L32

Liu, M. C., Dupuy, T. J., \& Allers, K. N. 2016, ApJ, 833, 96

Liu, M. C., Magnier, E. A., Deacon, N. R., et al. 2013, ApJL, 777, L20

Macintosh, B. A., Graham, J. R., Palmer, D. W., et al. 2008, Proc. SPIE, 7015, 701518

Malo, L., Doyon, R., Lafrenière, D., et al. 2013, ApJ, 762, 88

Mamajek, E. E., \& Bell, C. P. M. 2014, MNRAS, 445, 2169

Marocco, F., Andrei, A. H., Smart, R. L., et al. 2013, AJ, 146, 161

Martin, E. C., Mace, G. N., McLean, I. S., et al. 2017, ApJ, 838, 73

McGovern, M. R., Kirkpatrick, J. D., McLean, I. S., et al. 2004, ApJ, 600, 1020

McLean, I. S., Becklin, E. E., Bendiksen, O., et al. 1998, Proc. SPIE, 3354, 566

McLean, I. S., Graham, J. R., Becklin, E. E., et al. 2000, Proc. SPIE, 4008, 1048

McLean, I. S., McGovern, M. R., Burgasser, A. J., et al. 2003, ApJ, 596, 561

McLean, I. S., Prato, L., McGovern, M. R., et al. 2007, ApJ, 658, 1217

Miles, B. E., Skemer, A. J., Barman, T. S., Allers, K. N., \& Stone, J. M. 2018, ApJ, 869, 18

Murphy, S. J., Lawson, W. A., \& Bessell, M. S. 2013, MNRAS, 435, 1325

Newton, E. R., Charbonneau, D., Irwin, J., et al. 2014, AJ, 147, 20

Oppenheimer, B. R., Baranec, C., Beichman, C., et al. 2013, ApJ, 768, 24

Pöhnl, H., \& Paunzen, E. 2010, A\&A, 514, A81

Prato, L., Mace, G. N., Rice, E. L., et al. 2015, ApJ, 808, 12

Rice, E. L., Barman, T., Mclean, I. S., Prato, L., \& Kirkpatrick, J. D. 2010 ApJS, 186, 63

Riedel, A. R., Blunt, S. C., Lambrides, E. L., et al. 2017, AJ, 153, 95

Rodriguez, D. R., Zuckerman, B., Kastner, J. H., et al. 2013, ApJ, 774, 101

Rousselot, P., Lidman, C., Cuby, J.-G., Moreels, G., \& Monnet, G. 2000, A\&A, 354, 1134

Schlieder, J. E., Lépine, S., Rice, E., et al. 2012, AJ, 143, 114

Schmidt, S. J., West, A. A., Hawley, S. L., \& Pineda, J. S. 2010, AJ, 139, 1808

Seager, S., Deming, D., \& Valenti, J. A. 2009, ASSP, 10, 123

Shkolnik, E. L., Allers, K. N., Kraus, A. L., Liu, M. C., \& Flagg, L. 2017, AJ, 154, 69

Thompson, M. A., Kirkpatrick, J. D., Mace, G. N., et al. 2013, PASP, 125, 809

Torres, C. A. O., Quast, G. R., Melo, C. H. F., \& Sterzik, M. F. 2008, in Handbook of Star Forming Regions, Vol. II, ed. B. Reipurth (San Francisco, CA: ASP), 757

Weinberger, A. J., Anglada-Escudé, G., \& Boss, A. P. 2013, ApJ, 762, 118

Zapatero Osorio, M. R., Béjar, V. J. S., Miles-Páez, P. A., et al. 2014, A\&A, 568, A6

Zuckerman, B. 2019, ApJ, 870, 27

Zuckerman, B., Bessell, M. S., Song, I., \& Kim, S. 2006, ApJL, 649, L115

Zuckerman, B., \& Song, I. 2004, ARA\&A, 42, 685 